\title{
Genome-wide dynamics of replication timing revealed by in vitro models of mouse embryogenesis
}

\author{
Ichiro Hiratani, ${ }^{1}$ Tyrone Ryba, ${ }^{1}$ Mari Itoh, ${ }^{1}$ Joy Rathjen, ${ }^{2}$ Michael Kulik, ${ }^{3}$ Bernadett Papp, ${ }^{4}$ \\ Eden Fussner, ${ }^{5}$ David P. Bazett-Jones, ${ }^{5}$ Kathrin Plath, ${ }^{4}$ Stephen Dalton, ${ }^{3}$ Peter D. Rathjen, ${ }^{2}$ \\ and David M. Gilbert ${ }^{1,6}$
}

${ }^{1}$ Department of Biological Science, Florida State University, Tallahassee, Florida 32306, USA; ${ }^{2}$ Department of Zoology, University of Melbourne, Parkville, Victoria 3010, Australia; ${ }^{3}$ Department of Biochemistry and Molecular Biology, University of Georgia, Athens, Georgia 30602, USA; ${ }^{4}$ University of California Los Angeles, David Geffen School of Medicine, Department of Biological Chemistry, Jonsson Comprehensive Cancer Center, Molecular Biology Institute, Eli and Edythe Broad Center of Regenerative Medicine and Stem Cell Research, Los Angeles, California 90024, USA; ${ }^{5}$ Program in Genetics and Genome Biology, The Hospital for Sick Children, Research Institute, Toronto, Ontario M5G 1L7, Canada

\begin{abstract}
Differentiation of mouse embryonic stem cells (mESCs) is accompanied by changes in replication timing. To explore the relationship between replication timing and cell fate transitions, we constructed genome-wide replication-timing profiles of 22 independent mouse cell lines representing 10 stages of early mouse development, and transcription profiles for seven of these stages. Replication profiles were cell-type specific, with $45 \%$ of the genome exhibiting significant changes at some point during development that were generally coordinated with changes in transcription. Comparison of early and late epiblast cell culture models revealed a set of early-to-late replication switches completed at a stage equivalent to the postimplantation epiblast, prior to germ layer specification and down-regulation of key pluripotency transcription factors [POU5F1 (also known as OCT4)/NANOG/SOX2] and coinciding with the emergence of compact chromatin near the nuclear periphery. These changes were maintained in all subsequent lineages (lineage-independent) and involved a group of irreversibly down-regulated genes, at least some of which were repositioned closer to the nuclear periphery. Importantly, many genomic regions of partially reprogrammed induced pluripotent stem cells (piPSCs) failed to re-establish ESC-specific replication-timing and transcription programs. These regions were enriched for lineage-independent earlyto-late changes, which in female cells included the inactive $\mathrm{X}$ chromosome. Together, these results constitute a comprehensive "fate map" of replication-timing changes during early mouse development. Moreover, they support a model in which a distinct set of replication domains undergoes a form of "autosomal Lyonization" in the epiblast that is difficult to reprogram and coincides with an epigenetic commitment to differentiation prior to germ layer specification.
\end{abstract}

[Supplemental material is available online at http://www.genome.org. The microarray data from this study have been submitted to the NCBI Gene Expression Omnibus (http://www.ncbi.nlm.nih.gov/geo/) under accession no. GSE18019.]

Despite the rapidly growing collection of genome-wide chromatin profiles, higher-order chromosome organization and its developmental regulation in metazoans remain poorly understood. DNA replication provides an excellent forum with which to investigate these levels of chromosome organization (Hiratani and Gilbert 2009). The eukaryotic genome is comprised of large segments of chromosomes that are coordinately replicated at characteristic times during S-phase (Goren and Cedar 2003; MacAlpine et al. 2004; White et al. 2004; Norio et al. 2005; Woodfine et al. 2005; Schwaiger and Schubeler 2006; Karnani et al. 2007; Farkash-Amar et al. 2008; Hiratani et al. 2008; Desprat et al. 2009; Hiratani et al. 2009; Schwaiger et al. 2009). Early and late replicating domains show features of euchromatin and heterochromatin, respectively (Hiratani et al. 2009). For instance, in every multicellular system examined, early replication and transcription are positively correlated (MacAlpine and Bell 2005; Hiratani et al. 2009; references therein). Moreover, each of these segments occupies different subnuclear compartments depending on their replication time,

${ }^{6}$ Corresponding author.

E-mail gilbert@bio.fsu.edu; fax (850) 645-8447.

Article published online before print. Article and publication date are at http://www.genome.org/cgi/doi/10.1101/gr.099796.109. with early-replicating segments localized in the nuclear interior, while late-replicating sequences are enriched at the periphery of the nucleus and the nucleolus (Berezney et al. 2000).

Recent studies have provided direct evidence for extensive replication-timing changes during cell differentiation, but the extent to which these changes occur in different cell lineages and how they are coordinated with key cell fate decisions has not been addressed. Neural differentiation of mESCs is accompanied by replication-timing changes affecting $\sim 20 \%$ of the genome, with smaller differentially replicating domains consolidating into larger coordinately replicated units (Hiratani et al. 2008). In addition, $\sim 20 \%$ replication-timing differences were found between Drosophila embryonic versus wing disc cell lines (Schwaiger et al. 2009). Replication-timing changes are coordinated with transcription changes and rearrangements in subnuclear position (Williams et al. 2006; Hiratani et al. 2008), revealing a novel and unanticipated property of chromosome behavior during neural differentiation.

These findings motivated us to explore how this reorganization takes place during mouse embryogenesis. We addressed this by constructing genome-wide replication-timing profiles of a series of cell culture models derived from both ESCs and the embryo that represent distinct developmental stages and early embryonic tissues. We also addressed the stability of changes in replication 
Hiratani et al.

timing and transcription by reversing ESC differentiation, as well as analyzing partially and fully reprogrammed induced pluripotent stem cells (iPSCs) (Maherali et al. 2007). Altogether, we find that $45 \%$ of the genome experiences significant changes in replication timing between any cell types. Among them, we find that a significant number of early-to-late replication-timing changes occur in a lineage-independent manner, which are completed at a stage equivalent to the post-implantation epiblast, prior to germ layer specification and downregulation of key pluripotency transcription factors [POU5F1 (also known as OCT4)/NANOG/SOX2]. Despite small differences in transcription between early and late epiblast stages, replication domain organization clearly distinguished cell types beyond the late epiblast stage from earlier pluripotent cell types of the inner cell mass (ICM) and the early epiblast. Interestingly, subnuclear repositioning of replication-timing switching segments, as well as formation of compact chromatin at the nuclear periphery accompanied this transition from the early to late epiblast stages. Furthermore, autosomal segments that have completed a switch from early to late replication during the epiblast equivalent stage were particularly resistant to reprogramming, both in terms of replication and transcription, in a manner similar to the inactive $\mathrm{X}$ chromosome (Xi). Together, this study represents a detailed "fate map" of replication-timing regulation of the mouse genome during early embryonic stages. Furthermore, we propose that a set of lineage-independent early-to-late replication switches, completed at a time when the $\mathrm{Xi}$ also becomes late replicating, coincides with an epigenetic commitment to differentiation prior to germ layer specification, which then becomes difficult to reverse upon nuclear reprogramming.

\section{Results}

A synchronous differentiation system that recapitulates early mouse embryogenesis

To explore when replication domain reorganization (Hiratani et al. 2008) occurs during neural differentiation of ESCs, we employed a differentiation system that allows for the analysis of discrete intermediates (Fig. 1A). In this system, ESCs are differentiated in a conditioned medium designated MEDII for $9 \mathrm{~d}$ to form neural precursor cells (NPCs) (Rathjen et al. 2002; Rathjen and Rathjen

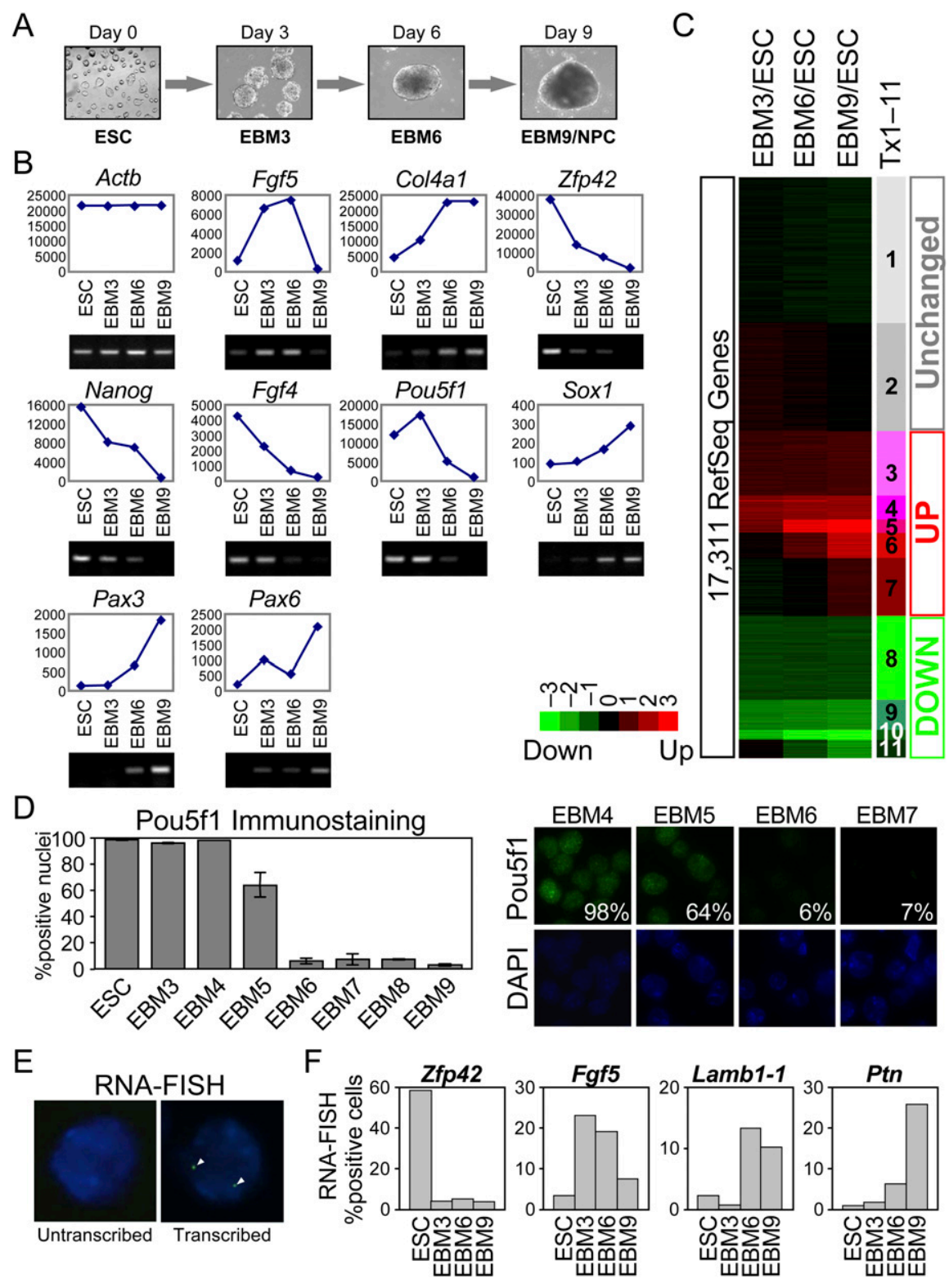

Figure 1. Synchronous differentiation of pluripotent ESCs to neurectoderm. (A) A 9-d neural differentiation scheme. See text for details. (B) Analyses of steady-state mRNA levels by microarrays (line charts; $y$-axis, arbitrary units) and reverse transcriptase (RT)-PCR (gel images) at 3-d intervals. See text for details. Actb (beta actin), loading control. (C) A heatmap showing 17,311 RefSeq genes present on the gene expression microarray, where each horizontal line represents a single gene. $\log _{2}$ transformed ratios of steady state mRNA levels in EBM3, EBM6, and EBM9 to those of ESC are displayed using the color code shown. Genes are ordered based on a K-means clustering into 11 expression clusters (Tx1-11) with distinct patterns, where gray $(\operatorname{Tx} 1-2)$, red (Tx3-7), and green (Tx8-11) color spectra show unchanged, up-regulated and down-regulated clusters, respectively. (D) Synchronous elimination of POU5F1 from nuclei. (Left) ESC and EBM3-9 were scored for POU5F1-positive nuclei. Similar results were obtained from three experiments and their average is shown. We scored over 330 nuclei for EBM5 and EBM6, while 54-302 were scored for others. Error bars represent standard error of the mean. (Right) Representative images from EBM4, EBM5, EBM6, and EBM7 are shown. Top, POU5F1 (green); bottom, DAPI (blue); Percentages, POU5F1-positive nuclei. (E,F) RNA-FISH reveals synchronous changes in transcription of tissue-specific genes. ( $E$ ) Representative images of cells with and without RNA-FISH signals (arrowheads). ( $F$ ) RNA-FISH analysis at 3-d intervals. One representative experiment for each gene is shown. At least 147 nuclei were scored for each state. Similar results were obtained in at least two replicate differentiation series. Note that even highly expressed genes are not $100 \%$ positive due to the probabilistic nature of interactions with the transcription machinery (Mitchell and Fraser 2008).

2003). Intermediates are designated EBM1-9, for embryoid bodies grown in MEDII for $\underline{1-9} \mathrm{~d}$. We previously reported replication profiles for the end points in this system, ESCs and NPCs (EBM9)

\section{Genome Research www.genome.org}


(Hiratani et al. 2008). Here, we analyzed day-3 (EBM3) and day-6 (EBM6) intermediates. Although this differentiation system has been shown to recapitulate neurectoderm formation in vivo (Rathjen et al. 2002), we further characterized each differentiation stage using gene expression microarrays (NimbleGen). The primitive ectoderm marker, $F g f 5$, is up-regulated in EBM3 and downregulated in EBM9, while an extracellular matrix component, Col4a1 (collagen, type IV, alpha 1), is up-regulated in EBM6 (Fig. 1B). $Z f p 42$ (previously known as Rex1), which marks the ICM but not the primitive ectoderm, is sharply down-regulated in EBM3, while other pluripotency factors, Nanog, Fgf4, and Pou $5 f 1$ (previously known as Oct4), showed later down-regulation in EBM6 (Fig. 1B). Neural markers, Sox1, Pax3, and Pax6, were only weakly active in EBM6, but increased their expression in EBM9 (Fig. 1B). Reverse transcriptase (RT)-PCR corroborated the microarray results (Fig. 1B). Furthermore, K-means clustering of 17,311 RefSeq genes (NCBI most well annotated genes present on the array) revealed 11 gene clusters with distinct patterns of up- and down-regulation in the differentiation intermediates, indicating that EBM3 and EBM6 represent intermediate states distinct from ESC, EBM9, or each other (Fig. 1C; Supplemental Table 1). Moreover, POU5F1 was sharply eliminated from the nuclei between days 5 and 6 , underscoring the distinction between EBM3 and EBM6, as well as the synchrony of differentiation (Fig. 1D). Ongoing transcription analysis by RNA-FISH with intronic probes, which reveals the dynamics of induction and repression because it circumvents the complications of mRNA processing and half-lives (Mitchell and Fraser 2008), further supported the synchrony (Fig. 1E,F). Zfp42 was sharply down-regulated by day 3, while Fgf5, Lamb1-1 (laminin B1 subunit 1; an extracellular matrix component), and Ptn (neurectoderm marker) were sharply up-regulated on days 3, 6, and 9, respectively (Fig. 1F), indicating synchronous cell fate transitions. Thus, EBM3 and EBM6 represent distinct intermediates. Expression profiles are consistent with EBM3 resembling primitive ectoderm (Rathjen et al. 1999), while EBM6 resembles definitive ectoderm (Rathjen et al. 2002). Taken together, we conclude that EBM3 and EBM6 provide the opportunity to examine distinct intermediate stages of early neural development in mice.

\section{Early-to-late precede late-to-early replication-timing changes}

To construct genome-wide replication-timing profiles of EBM3 and EBM6, cells were labeled with BrdU, separated into early and late S-phase fractions by flow cytometry, and BrdU-substituted DNA from early and late S-phase populations was immunoprecipitated, differentially labeled, and cohybridized to a mouse whole-genome oligonucleotide microarray (NimbleGen). This generates a replication-timing ratio $\left[=\log _{2}\right.$ (Early/Late) for each of the tiled probes positioned every 5.8 kilobases $(\mathrm{kb})$ throughout the entire mouse genome (Hiratani et al. 2008). Replicates (dye-switched) showed high correlation (Supplemental Fig. 1) and were averaged to generate loess-smoothed curves along the chromosomes, as well as replication domain maps, using a segmentation algorithm (Fig. 2A), as described previously (Hiratani et al. 2008). Data sets were confirmed by PCR analysis of 18 genes (Supplemental Fig. 2). Microarrays with $5.8-\mathrm{kb}$ probe densities generate an almost indistinguishable profile as those with probes every 100 base pairs (Hiratani et al. 2008), allowing reliable genome-wide replication-timing analysis on a single oligonucleotide chip. Moreover, similar results are obtained with methods that evaluate S-phase copy number, demonstrating that early-late fractionation does not introduce a temporal bias (Hiratani et al. 2008). All data sets generated in this study are graphically displayed and downloadable on our website: http:// www.replicationdomain.org (Weddington et al. 2008).

Pearson's $R^{2}$-values for pairwise comparisons to ESC replication profile showed continuous changes during differentiation (Fig. 2B). However, changes did not occur uniformly. First, by visual inspection, chromosomal domains that changed from early to late (EtoL: defined as any significant shift to later replication) did so primarily during the ESC-EBM6 transition (Fig. 2C), whereas domains that changed from late to early (LtoE) did so primarily during the EBM3-EBM9 transition (Fig. 2D). Second, while the number of replication domains decreased gradually via replication domain consolidation (data not shown), as previously described (Hiratani et al. 2008), the correlation to isochore GC/LINE-1 content sharply improved from EBM3 to EBM6 (Fig. 2E; Supplemental Fig. 3). To comprehensively evaluate the temporal differences between EtoL and LtoE changes, we analyzed the replication timing of 18,679 RefSeq genes. Loess-smoothed replication-timing ratios at their transcription start sites were calculated as described (Hiratani et al. 2008), and genes were categorized into 20 clusters by K-means clustering (Fig. 2F; Supplemental Table 1; kinetics in Fig. 2G,H; Supplemental Fig. 4). Among the 20 clusters, we identified clusters $9-12$ (10\% of all genes) and 13-16 (9\%) as those that shift their timing earlier (LtoE) and later (EtoL), respectively (Fig. 2F). Line charts of mean ratios clearly showed that LtoE changes occurred primarily during the EBM3-EBM9 transition (Fig. 2G), while EtoL changes occurred during the ESC-EBM6 transition (Fig. $2 \mathrm{H})$. These results demonstrate that the majority of EtoL changes precede LtoE changes and suggest the hypothesis that EtoL changes are associated with loss of pluripotency, while LtoE changes occur later, during lineage commitment.

\section{Subnuclear repositioning associated with replication-timing changes occur during the EBM3-EBM6 transition, in parallel with chromatin fiber reorganization}

During differentiation of ESCs to NPCs, chromosomal segments that change replication timing also exhibit spatial repositioning (Hiratani et al. 2008). To assess when this spatial reorganization occurs, we measured the radial subnuclear positioning of eight loci by three-dimensional (3D) DNA fluorescence in situ hybridization (FISH) in EBM3 and EBM6. Data distribution is displayed using box plots (Fig. 3) or cumulative frequency plots (Supplemental Fig. 5). ESC and EBM9 data (Hiratani et al. 2008), as well as replicationtiming profiles, are shown alongside for comparison (Fig. 3). Interestingly, all three EtoL loci analyzed exhibited significant repositioning toward the nuclear periphery during the EBM3-EBM6 transition, while other 3-d intervals showed little or no changes (Fig. 3A, Zfp42, Rex2, and Dppa2; cf. P-values; Fig. 3D shows exemplary FISH images of Dppa2). All three LtoE loci analyzed also exhibited the most significant repositioning during the EBM3-EBM6 transition, moving away from the nuclear periphery (Fig. 3B, Ptn, Ephb1, and Akt3). Two control genes that were down-regulated but remained early replicating maintained their internal position during differentiation (Fig. 3C, Pou5f1 and Nanog). Thus, the EBM3-EBM6 transition was accompanied by spatial repositioning of all chromosomal segments tested that changed replication timing. Unfortunately, DNA-FISH, even when carried out at a larger scale, would still only query a fraction of the genome. However, in both ESCs and differentiated cell types, DNA synthesis takes place almost exclusively in the interior of the nucleus throughout the first half of S-phase, and then dramatically transitions to the periphery in middle (mid) S-phase (Panning and Gilbert 2005; Wu et al. 
A

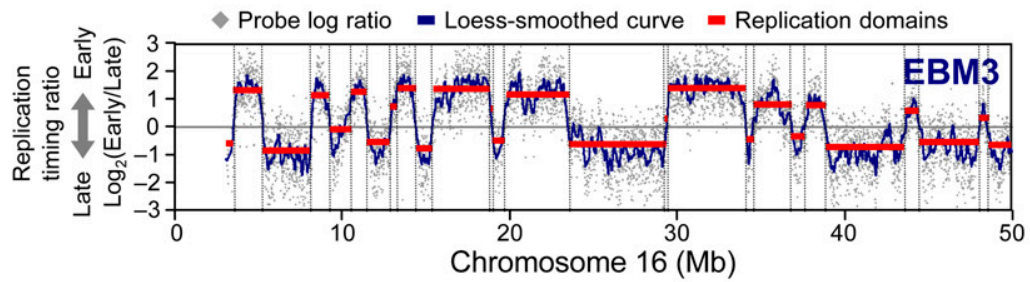

B

\begin{tabular}{lccc}
$R^{2}$ & EBM3 & EBM6 & EBM9 \\
\hline D3ESC & 0.78 & 0.73 & 0.67 \\
EBM3 & & 0.85 & 0.64 \\
EBM6 & & & 0.84 \\
\hline
\end{tabular}

C

\begin{tabular}{crrrr} 
E $\begin{array}{c}R^{2}, \text { vs } \\
\text { Replication } \\
\text { Timing }\end{array}$ & ESC & EBM3 & EBM6 & EBM9 \\
\hline$\%$ GC & 0.26 & 0.29 & 0.50 & 0.50 \\
$\%$ LINE-1 & 0.18 & 0.26 & 0.40 & 0.40 \\
\hline
\end{tabular}

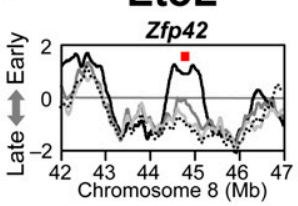

D LtoE

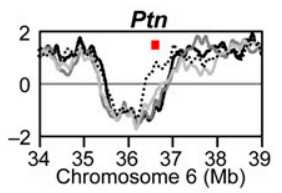

Rex2
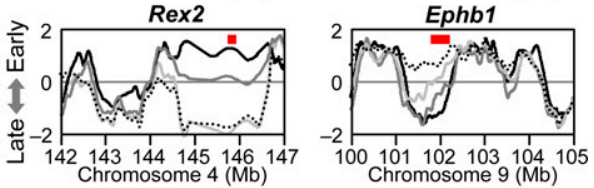

Dppa2
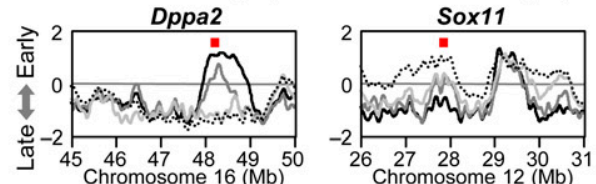

F
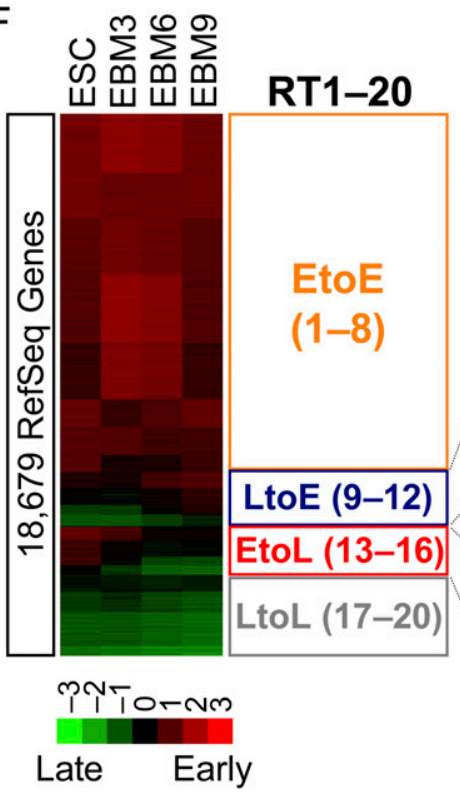

Early
RT1-20

(1-8)

G

G
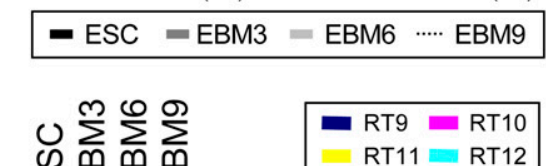

邑思

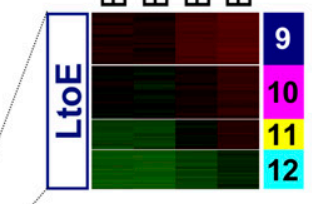

$\mathrm{H}$

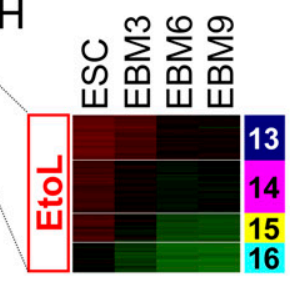

2005). This global association implies that subnuclear repositioning of our limited sampling likely translates to most loci whose replication-timing changes traverse the mid S-phase (Hiratani et al. 2009). Consistently, all loci reposition only when they traverse mid-late S-phase (Fig. 3A,B; highlighted in pink).

To address the potential for larger scale spatial reorganization of chromatin during the EBM3-EBM6 transition, we took an alternative approach to analyze nuclear chromatin conformation directly in ESCs, EBM3, EBM6, and EBM9 using electron spectroscopic imaging (ESI). ESI, also referred to as energy-filtered transmission electron microscopy (EFTEM), is an analytical technique that can generate high-resolution elemental maps of conventionally prepared cells or tissues, allowing a finer delineation of chromatin organization than conventional electron microscopy (Bazett-Jones et al. 2008). By ESI, ESC nuclei can be described as a relatively uniform meshwork of $10 \mathrm{~nm}$ chromatin fibers with a low degree of chromatin compaction along the nuclear envelope or throughout the nucleoplasm (Fig. 3E). EBM3 showed a landscape very similar to ESCs. However, in EBM6, a dramatic accumulation of compact chromatin was evident near the nuclear periphery, and the boundaries of these compact chromatin domains became further sharpened in EBM9 (Fig. 3E). Thus, the EBM3-EBM6 transition is accompanied by a dramatic reorganization of chromatin conformation. This is coincident with completion of EtoL changes, repositioning of EtoL loci toward the nuclear periphery, and alignment of replication domains to isochores, leading us to hypothesize this transition as a period of extensive genome reorganization.

Changes in replication-timing and transcription reveal an overall coordination of directionality but not kinetics

Figure 2. Kinetics of replication-timing changes during neural differentiation of ESCs. (A) Replicationtiming profiling. Exemplary profile of EBM3 is shown. (Gray dots) Probe log ratios [ $\log _{2}$ (Early/Late)] along chromosome 16. A local polynomial smoothing (loess) curve is overlaid (blue). Replication domains (red lines) and their boundaries (dotted lines) identified by segmentation are also overlaid (Hiratani et al. 2008). (B) Pearson's $R^{2}$-values for pairwise comparisons of replication timing. Smoothed data for all probes were used for the calculation. $(C, D)$ Overlaid replication-timing profiles of ESC, EBM3, EBM6, and EBM9, at selected EtoL domains $(C)$ and LtoE domains $(D)$, using the color code at the bottom. Representative genes within the domains are shown with their chromosomal positions in red squares. ( $E$ ) Pearson's $R^{2}$-values are shown for the relationship between average replication-timing ratios of replication domains vs. their \%GC and \%LINE-1 values in ESC, EBM3, EBM6, and EBM9. (F) A heatmap showing replication-timing ratios $\left[=\log _{2}\right.$ (Early/Late)] of 18,679 RefSeq genes based on the microarrays using the color code shown. Genes are ordered based on a K-means clustering into 20 replication-timing clusters with distinct patterns. Orange, blue, red, and gray boxes represent EtoE (RT1-8), LtoE (RT9-12), EtoL (RT13-16), and LtoL (RT17-20) clusters, respectively (kinetics in G, $H$ and Supplemental Fig. 4). ( $G, H)$ Magnified views of LtoE ( $G$, RT9-12) and EtoL (H, RT13-16) clusters, respectively. Line charts show mean replication-timing ratios of each cluster.

To determine how changes in replication timing and transcription are temporally coordinated, we analyzed the relationship between replication timing and transcription of 17,311 RefSeq genes. Specifically, we cross-tabulated the K-means clusters of replication timing (Fig. 2F) and transcription (Fig. 1C). The results were graphically displayed (Fig. 4A; Supplemental Table 1), with each of the 20 replication-timing clusters, referred to as RT1-20 (Fig. 2F), presented as a stacked bar graph showing

\section{Genome Research \\ www.genome.org}


A

B

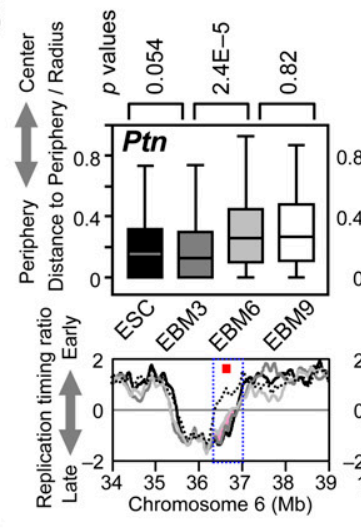

D

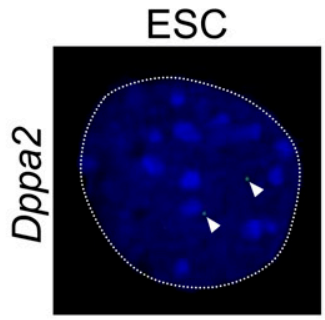

E

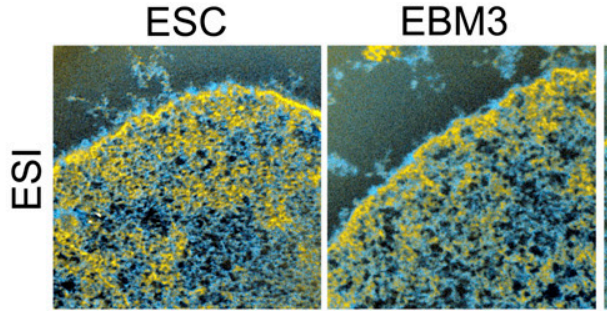

C
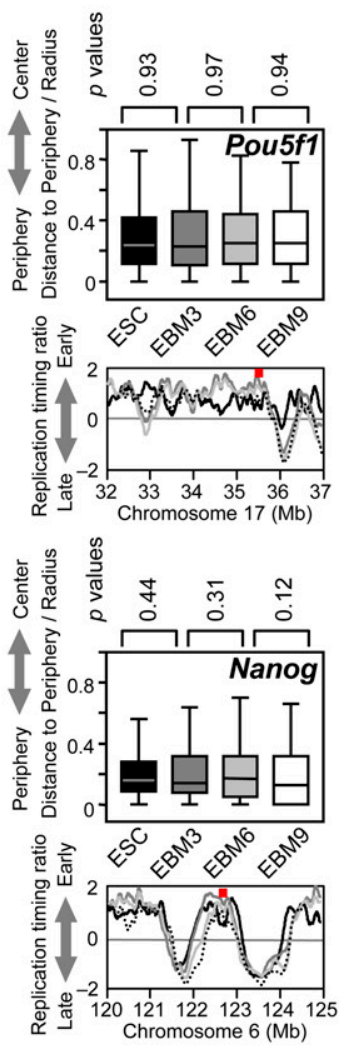

Chromosome 1 (Mb)

EBM6

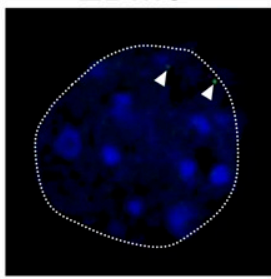

EBM6

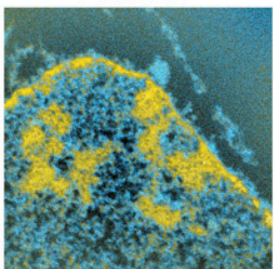

\section{EBM9}

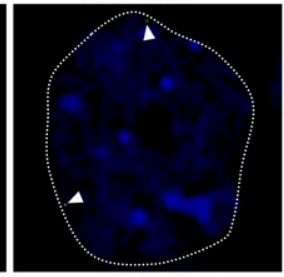

EBM9

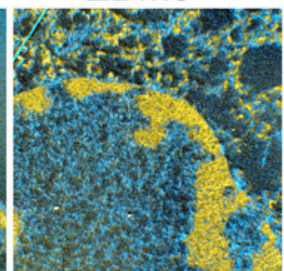

Figure 3. Subnuclear repositioning associated with replication-timing changes occurs during the EBM3-EBM6 transition, in parallel with chromatin fiber reorganization. ( $A-C$ ) Analysis of subnuclear positions of eight genomic regions by 3D DNA-FISH in EBM3 and EBM6. ESC and EBM9 data (Hiratani et al. 2008) are also shown for comparison. Box plots show the distribution of relative radial distance to the nuclear periphery, where 0 and 1 represents the periphery and the center of the nucleus, respectively. Horizontal bars represent the 10th, 25th, 50th (median), 75th, and 90th percentiles. P-values were obtained from a two-sample Kolmogorov-Smirnov test. Below the box plots are the overlays of the four replication-timing profiles (using the color code at the top) with the probe gene positions (red squares). All EtoL domains ( $A ; Z f p 42$, Rex2, and Dppa2) and LtoE domains ( $B ;$ Ptn, Ephb1, and Akt3) analyzed make the most significant movement toward and away from the nuclear periphery, respectively, during the EBM3-EBM6 transition. During this period, replication-timing changes traverse the mid-late S-phase (pink shades), which is when a dramatic interior to peripheral transition in the spatial patterns of DNA replication foci occurs (Hiratani et al. 2009). In contrast, two EtoE domains (C; Pou5f1 and Nanog) do not change subnuclear positioning or replication time. Comparable results were obtained from two to four biological replicates, and the sum of all experiments is shown. Seventy-one to 223 FISH signals were measured per state. The identical data sets are also displayed using cumulative frequency plots (Supplemental Fig. 5). (D) Representative FISH images of Dppa2. Dotted lines represent the rim of nuclear DAPI signals. Arrowheads point to green FISH signals. (E) Electron spectroscopic imaging (ESI) analysis of nuclei from ESC, EBM3, EBM6, and EBM9. Relative levels of phosphorus and nitrogen levels were used to delineate chromatin (yellow) vs. protein and ribonucleoprotein (blue) (Bazett-Jones et al. 2008). 
A

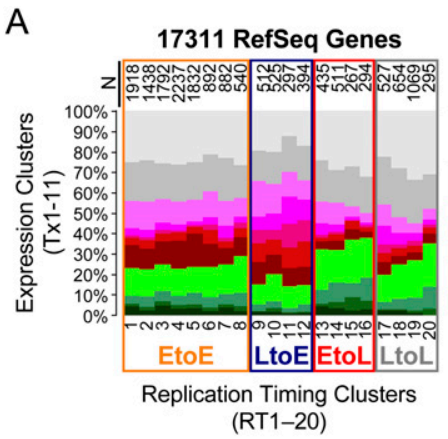

C

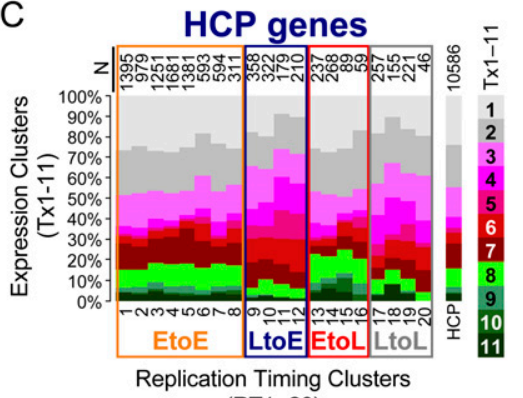

(RT1-20)

E

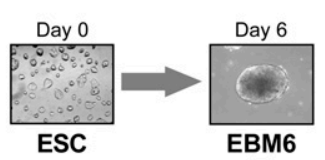

EBM6R
$\mathrm{F}$

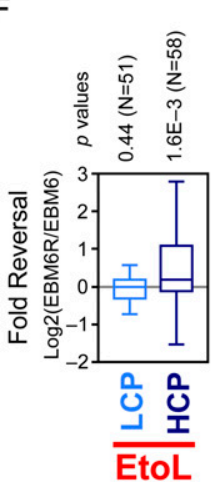

B

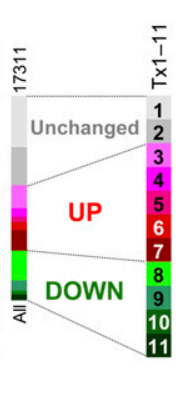

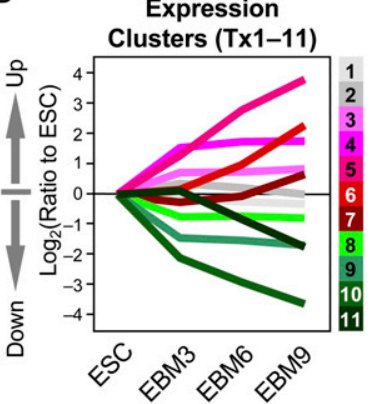

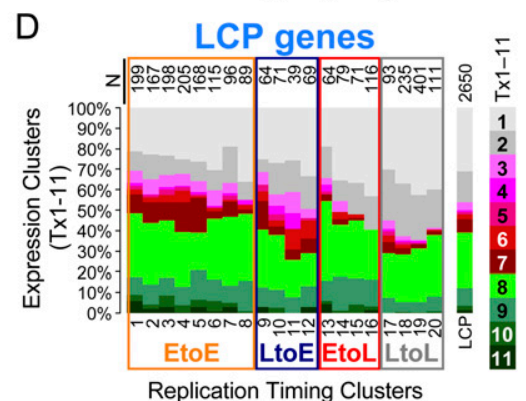

(RT1-20)

G

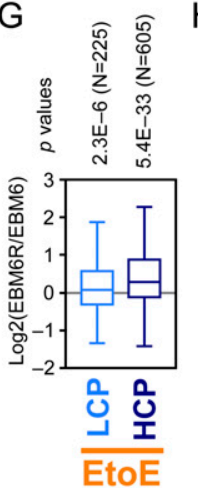

Figure 4. Relationship between replication-timing changes, transcription changes, and their reversibility $(A)$ Cross-tabulation of K-means clusters of replication timing and transcription. All 17,311 RefSeq genes present on the gene expression array were analyzed. Each of the 20 replication-timing clusters, RT1-20 (Fig. 2F), were presented as a stacked bar consisting of the 11 expression clusters, Tx1-11 (Fig. 1C). N, number of genes in RT1-20. (B) Line chart showing the kinetics of transcription changes of Tx1-11 during differentiation. $\log _{2}$ transformed ratios to ESC levels are plotted. $(C, D)$ The same crosstabulation graph as in A, for 10,586 HCP genes $(C)$ and $2650 \mathrm{LCP}$ genes $(D)$. HCP and LCP classification is based on Mikkelsen et al. (2007). (E) A scheme for the reversal of differentiation. EBM6R is a condition in which EBM6 cells were placed back into ESC medium containing LIF and cultured for $6 \mathrm{~d}$. $(F, G)$ Box plots showing the reversibility of different classes of genes. $\log _{2}$ transformed expression level in EBM6R relative to EBM6 is plotted. Twofold down-regulated genes during the ESC-EBM6 transition were selected from the down-regulated expression clusters, Tx8-11. Among them, genes in the EtoL clusters (RT13-16) are presented in $F$, while genes in the EtoE clusters (RT1-8) are presented in G. $P$-values were obtained from a two-tailed $t$-test for comparison of two paired groups (i.e., EBM6R vs. EBM6). $(H)$ LCP is overrepresented in EtoL genes that are not reversed in EBM6R. Twofold down-regulated EtoL and EtoE genes that did not show reversal (i.e., EBM6R/EBM6 $<1$ ) were calculated for their LCP-to-HCP ratio. Note that there are four times as many HCPs as LCPs in the genome (=All genes). Numbers of genes in each category are 49 (EtoL) and 313 (EtoE). A $P$-value was obtained from a $\chi^{2}$ test.

percentages of the 11 expression clusters, Tx1-11 (Fig. 1C; kinetics of each cluster in Fig. 4B). We found that EtoE clusters (RT1-8) show a comparable rate of up- and down-regulation and a similar distribution of expression clusters, suggesting that transcriptional regulation is independent of precisely when they replicate during the first half of S-phase (kinetics of each cluster in Supplemental Fig. 4) (Hiratani et al. 2009). In contrast, LtoE clusters (RT9-12) clearly showed a tendency for up-regulation (Tx3-7; red color
$\mathrm{H}$

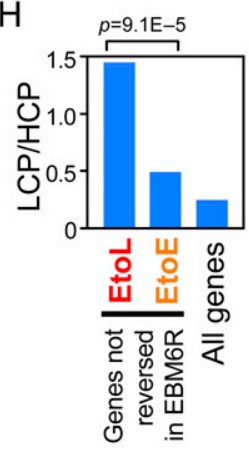

spectrum assigned; $P=1.5 \times 10^{-51}, \chi^{2}$ test), while EtoL clusters (RT13-16) showed much lower ratio of up-regulation (Tx3-7) and a higher ratio of down-regulation (Tx8-11; green color spectrum assigned; $P=2.8 \times 10^{-17}, \chi^{2}$ test). However, replication-timing changes could precede, follow, or occur within the same time window as transcriptional changes, showing no consistent kinetic relationship. Interestingly, the period of extensive genome reorganization, the EBM3EBM6 transition, showed the least transcriptional change (Supplemental Fig. 6).

\section{Upon early-to-late replication-timing changes, down-regulated low $\mathrm{CpG}$ density promoters become difficult to up-regulate}

Figure $4 \mathrm{~A}$ also revealed a considerable number of exceptional genes that are upregulated, even during EtoL replicationtiming switches. We previously suggested that genes with high versus low $\mathrm{CpG}$ density promoters (HCP vs. LCP genes), which show different modes of regulation (Mikkelsen et al. 2007; Weber et al. 2007), behave differently upon changes in replication timing (Hiratani et al. 2008). We revisited this comprehensively utilizing the same cross-tabulation method as in Figure 4A, plotting HCP (Fig. 4C) and LCP (Fig. 4D) genes separately. Strikingly, HCP genes showed a strong tendency toward up-regulation regardless of replication timing (Fig. 4C), whereas LCP genes were rarely up-regulated within EtoL or LtoL clusters (Fig. 4D). In fact, the latest replicating EtoL cluster (RT16) did not contain any up-regulated LCP genes (Fig. 4D). These results suggest that LCP gene activation is considerably less compatible with late replication than HCP gene activation.

To investigate whether late replication is related to the responsiveness of silent LCP genes, we devised an assay to assess reversibility of LCP genes within EtoL clusters. Briefly, we returned EBM6 to ESC medium containing LIF for $6 \mathrm{~d}$ (Fig. 4E, EBM6R) and profiled gene expression using microarrays. We then measured the expression level in EBM6R, relative to EBM6, for genes that were down-regulated by over twofold during the ESC-EBM6 transition. EBM6R did not exhibit a complete reversal, indicating that EBM6 cells are committed and cannot fully revert back to the ESC state. Nevertheless, partial up-regulation of genes that became downregulated during the ESC-EBM6 transition allowed us to assess the relationship of replication-timing changes and CpG density to reversibility. Results revealed that down-regulated LCP genes that had undergone EtoL replication-timing switches were not 
significantly up-regulated, whereas EtoL HCP genes showed partial yet statistically significant reversal of transcription levels (Fig. 4F, cf. box plot positions relative to 0 ). In contrast, EtoE genes showed partial reversibility regardless of promoter CpG content (Fig. 4G), suggesting that an early-replicating environment is more permissive for LCP gene up-regulation. Consistently, when we focused only on those genes that became irreversibly downregulated after an EtoL switch, LCP genes were highly enriched over HCP genes (Fig. 4H). RNA-FISH analysis of nascent transcripts for three EtoL genes confirmed their nonresponsiveness to ESC medium in EBM6R, while two EtoE genes were more easily upregulated (Supplemental Fig. 7). Gene expression reversal cannot be explained by the expansion of residual pluripotent cells in the differentiated population, as this would appear as uniform partial up-regulation of all promoter classes. While it is known that CpGs in LCPs are mostly methylated in somatic cells, their methylation does not appear to preclude their transcriptional activity and they are not preferential targets of de novo methylation (Weber et al. 2007). Thus, we believe it is unlikely that DNA methylation of LCPs, even if it had occurred during the ESC-EBM6 transition, can account for the observed loss of reversibility. Together, these data suggest that replication-timing switches are associated with the transcriptional responsiveness of specific types of promoters while the activity of other promoters is unrelated to replication timing.

\section{Lineage-independent early-to-late replication-timing} changes and subnuclear repositioning are completed at the post-implantation epiblast stage

To place replication and transcription changes into the context of mouse embryogenesis, we constructed replication-timing profiles of two additional cell types that model early embryogenesis, EPL cells and EpiSCs. EPL cells are early primitive ectoderm-like cells derived from ESCs in adherent culture in a conditioned medium, which can be maintained for multiple generations (Rathjen et al. 1999) and show similarity in gene expression pattern to EBM3 (Supplemental Fig. 8). By marker gene expression, EPL cells and EBM3 resemble the early post-implantation epiblast at embryonic day (E) 4.75-5.25 (Pelton et al. 2002). EpiSCs are derived from later post-implantation epiblast at E5.5 (Brons et al. 2007; Tesar et al. 2007), but retain a gene expression profile similar to EPL/EBM3 cells (Fig. 5A; Supplemental Fig. 8) and still express the pluripotency circuitry transcription factors (POU5F1/NANOG/SOX2), which we confirmed by immunofluorescence (Fig. 5B), as well as gene expression microarrays (Supplemental Fig. 8). EpiSCs and EPL cells are both capable of generating cells of all three germ layers in vitro but contribute very poorly, if at all, to the formation of chimeric mice (Rathjen et al. 1999; Brons et al. 2007; Tesar et al. 2007). Despite their resemblance, EPL cells readily revert back to the ESC state by culture in ESC medium containing LIF (Rathjen et al. 1999), upon which they can contribute to chimeric mice (Rathjen et al. 1999), whereas EpiSCs cannot (Brons et al. 2007; Tesar et al. 2007). In fact, generating iPSCs from EpiSCs is as inefficient $(0.1 \%-1 \%)$ as from other somatic cell types (Guo et al. 2009). This suggests that EpiSCs have progressed beyond an as-yet unidentified epigenetic barrier that is difficult to overcome upon nuclear reprogramming.

To compare EPL and EpiSCs with previously profiled cell types (Hiratani et al. 2008), we expressed replication profiles as numeric vectors of 10,974 average replication-timing ratios of nonoverlapping 200-kb windows across the genome for each cell line
(Supplemental Table 2). Comparison of 12 cell line profiles identified significant replication-timing differences across $15 \%$ of the genome (Fig. 5C). As shown, no clusters traversed the entire S-phase, from very early to very late S-phase or vice versa (Fig. 5C). Hierarchical clustering analysis clearly distinguished committed ectodermal cell types from ICM/early epiblast cell types, ESCs, EBM3, and EPL cells (Fig. 5C). Surprisingly, EpiSCs (Tesar et al. 2007) formed a branch together with the committed cell types. The distinction was particularly evident in the EtoL changes that were completed in EpiSCs, but not in EPL cells (Fig. 5C). Indeed, at several exemplary chromosomal regions, EPL cells clearly resembled EBM3, while EpiSCs clearly resembled the ectodermcommitted EBM6 (Supplemental Fig. 9).

We next determined whether subnuclear repositioning of EtoL loci was evident in EpiSCs versus EPL cells. By two-color twodimensional (2D) DNA-FISH using Pou5f1 as an internal control that does not reposition (Figs. 3C, 5D), Zfp42, Rex2, and Dppa2, which have completed EtoL changes in EpiSCs, but not EPL cells, clearly moved toward the nuclear periphery in EpiSCs, but not EPL cells (Fig. 5E-I; Supplemental Fig. 10). Furthermore, accumulation of compact chromatin near the nuclear periphery was also evident in EpiSCs by ESI (K Ahmed and DP Bazett-Jones, unpubl.). Meanwhile, the expression of POU5F1 in EpiSCs, but not EBM6 (Figs. $1 \mathrm{D}, 5 \mathrm{~B})$ and its pluriptotency clearly place EpiSCs upstream from EBM6. Hence, the completion of EtoL changes, their repositioning toward the nuclear periphery and the accumulation of compact chromatin near the periphery provide a concrete distinction between EpiSCs and EPL cells. Moreover, this suggests extensive genome reorganization prior to germ layer formation, at a time equivalent to the post-implantation epiblast. This coincides with EpiSCs becoming incompetent to easily revert back to the ESC state, while EPL cells, which have only partially changed replication timing, but have not completed the reorganization process, can easily revert back to the ESC state (Rathjen et al. 1999).

\section{Replication-timing changes across different lineages collectively affect nearly half the genome and include a set of lineage-independent early-to-late changes}

Important unanswered questions concern the extent of replication-timing changes in separate lineages of differentiated cells and whether such changes revert later during development. Furthermore, if there are stable lineage-independent replication-timing changes that occur in the epiblast, such changes should be found in common between separate lineages. To address these points, we profiled four additional cell types: Goosecoid(Gsc)+Sox17- early mesoderm and Gsc+Sox17+ endoderm cells derived from ESC differentiation (Yasunaga et al. 2005), as well as late mesodermal cell types derived from embryos, mouse embryonic fibroblasts (MEF) and fetal myoblasts (characterization of cells in Supplemental Figs. 11, 12). Replication profiles were expressed as a vector consisting of replication-timing ratios of $200-\mathrm{kb}$ segments genomewide, as in Figure 5C. Hierarchical clustering of 17 profiles (Fig. 6A, dendrogram) confirmed the epigenetic separation of EpiSCs from ICM/early epiblast cell types, with the former more related to committed germ layer cell types of the early embryo. The most extensive replication-timing changes distinguished late mesoderm cell types from early embryonic cell types (Fig. 6A), demonstrating that changes continue to occur after germ layer commitment. Notably, replication domain consolidation (Hiratani et al. 2008) was specific to ectoderm and was not evident in mesoderm and endoderm or in EpiSCs (data not shown). 
A Expression profile comparison (17311 RefSeq genes)

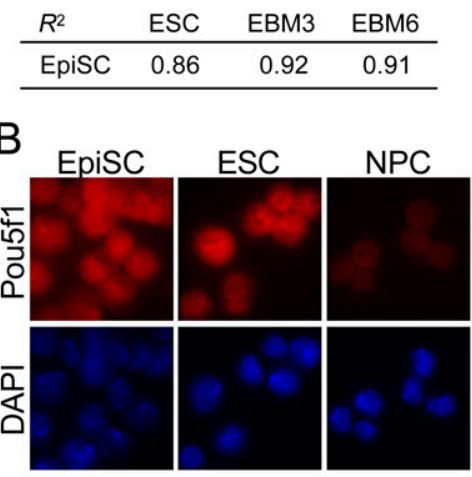

C

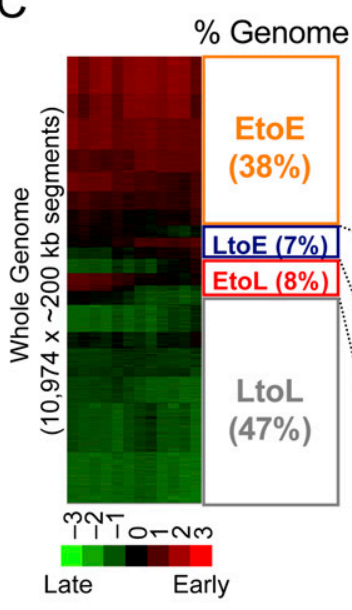

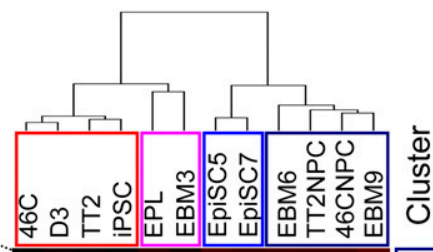

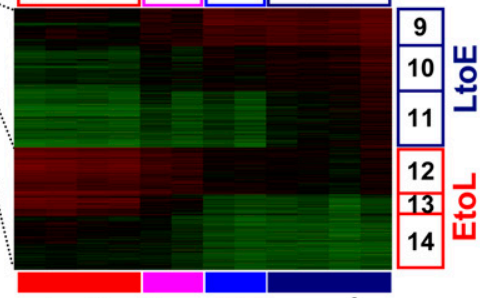

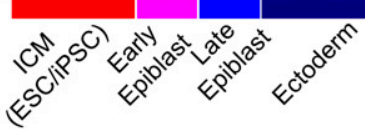

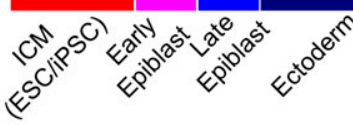

D

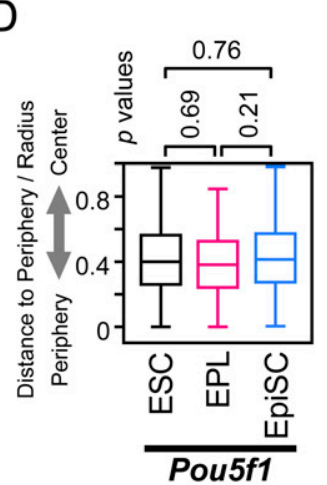

$\mathrm{H}$

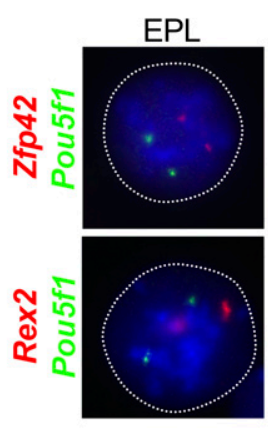

E

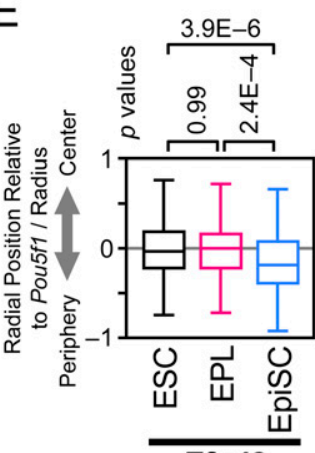

F

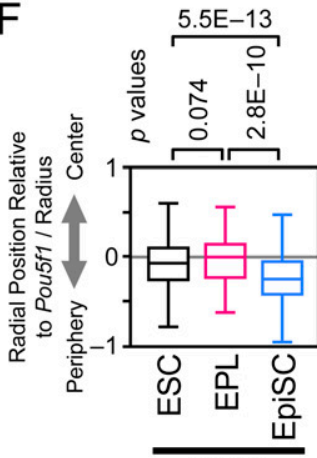

$\operatorname{Rex} 2$

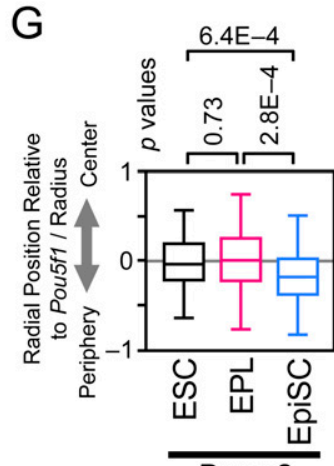

Dppa2

Zfp42

\section{EpisC}

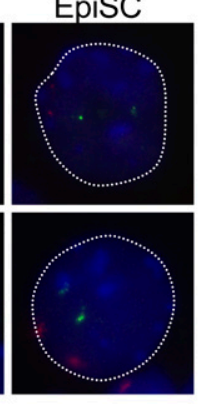

I

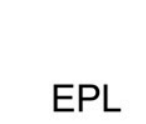

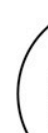

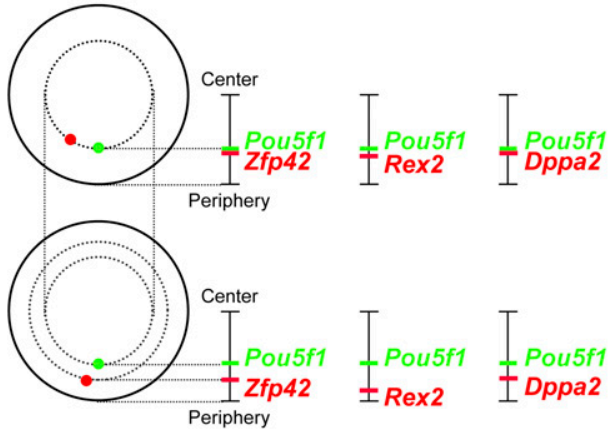

Figure 5. Lineage-independent replication-timing changes are completed at the EpiSC stage, coincident with spatial repositioning of early-to-late domains. (A) Pearson's $R^{2}$-values for pairwise comparisons of gene expression microarray profiles of 17,311 RefSeq genes. EpiSC shows the highest correlation to EBM3. (B) Immunostaining confirms POU5F1 expression in EpiSC5 (89\% positive; 88\% for EpiSC7 [data not shown]). The identical EpiSC batches were used for replication analysis. ESCs and NPCs are positive and negative controls, respectively. (C) Hierarchical clustering of 12 cell lines. The whole genome was divided into 10,974 200-kb segments and their average replication-timing ratios were compared between cell lines. (Left) A wholegenome heatmap and the percentages of segments that stay early (EtoE), stay late (LtoL), shift earlier (LtoE), and shift later (EtoL), based on a K-means clustering $(K=20)$. LtoE and EtoL clusters were defined as those that show a replication-timing differential of above 1.0 between any cell types. With this stringent cutoff, $15 \%$ of the genome exhibited differences (as opposed to $20 \%$ with a less stringent cutoff; Hiratani et al. 2008). Clusters 9-14 correspond to LtoE and EtoL segments used for hierarchical clustering on the right. The dendrogram draws a clear distinction between EPL/EBM3 and EpiSCs, which corresponds to early and late epiblast stages, respectively. Cell lines not described in this study are from Hiratani et al. (2008). ( $D-I$ ) Two-color 2D DNA-FISH in ESCs, EPL cells, and EpiSCs (EpiSC7 line). (D) Pou5f1, which is early replicating in all three cell lines, maintains its internal positioning. Box plots show the distribution of relative radial distance to the periphery, where 0 and 1 represents the periphery and the center of the nucleus, respectively. Relative radial distance medians were 0.40 (ESC), 0.38 (EPL), and 0.42 (EpiSC). N=222-430. (E-G) Subnuclear positioning of EtoL loci (Zfp42, Rex2, and Dppa2) relative to Pou5f1. Radial distance of Pou5f1 loci to the nuclear periphery was subtracted from that of $Z f p 42(E)$, Rex2 ( $F$ ), or Dppa2 (G) loci for all four combinations of allele pairs within a given nucleus, divided by the radius and their distribution was plotted as box plots. Most peripheral, identical, and most internal positioning of these loci relative to Pou5f1 are represented by $-1,0$ and 1 , respectively. $P$-values were obtained from a two-sample Kolmogorov-Smirnov test. All three EtoL loci exhibited significant repositioning toward the periphery in EpiSCs, but not others. $N=120-384$. The identical data sets are also shown using cumulative frequency plots (Supplemental Fig. 10). $(H)$ Representative images of 2D DNA-FISH. (I) Summary of 2D DNA-FISH. Positions shown for each locus (red, EtoL loci; green, Pou5f1) represent the median relative radial distance to the nuclear periphery, showing repositioning of EtoL loci toward the periphery in EpiSCs, but not EPL cells. In EPL cells, median values were: Zfp42 (0.40), Rex2 (0.30), and Dppa2 (0.42). In EpiSCs, median values were: Zfp42 (0.23), Rex2 (0.11), and Dppa2 (0.27).

\section{Genome Research}


A

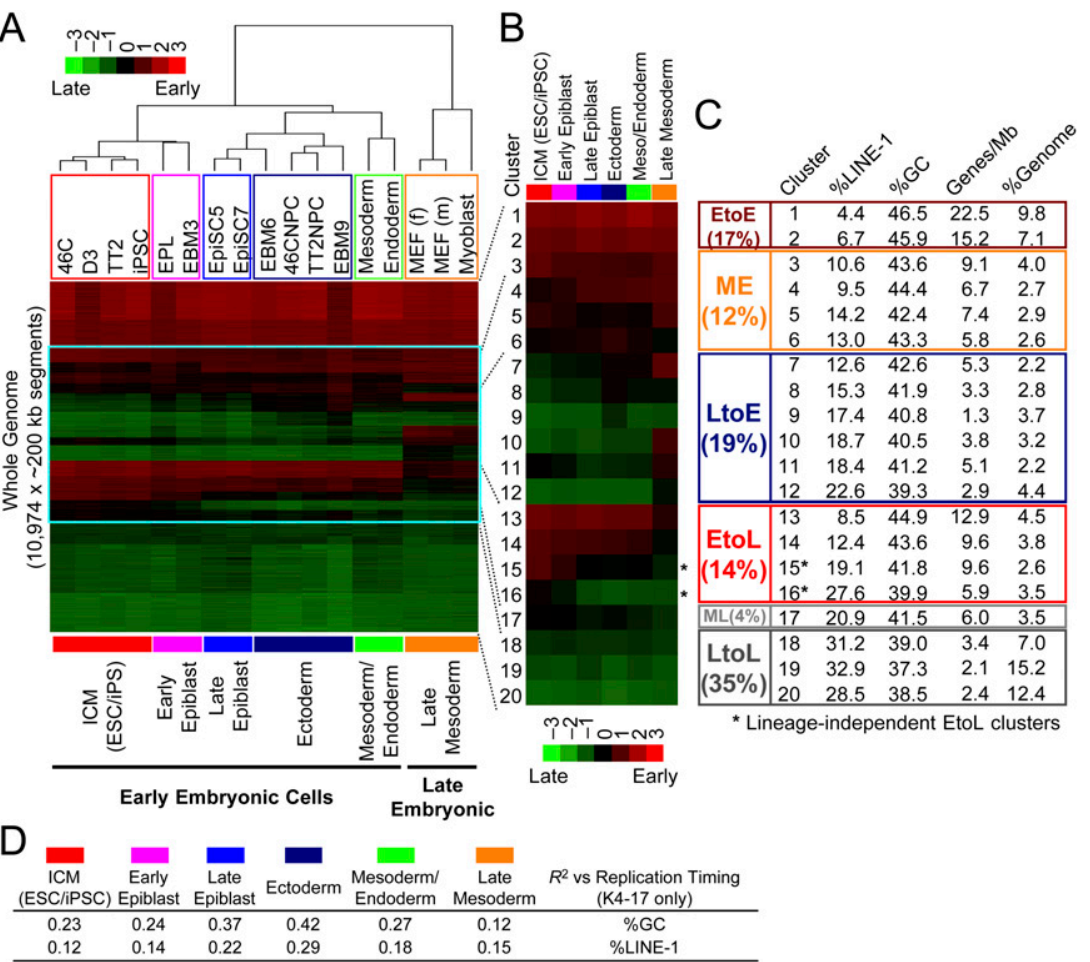

Figure 6. Replication-timing changes across different lineages collectively affect nearly half the genome and include a set of lineage-independent early-to-late changes. $(A)$ Hierarchical clustering with the addition of Gsc+Sox17- mesoderm, Gsc+Sox17+ endoderm, embryonic fibroblasts (f, female; $m$, male), and fetal myoblast cells. Their characterization is provided in Supplemental Figures 11 and 12 . $\mathrm{K}$-means clustering was applied first $(K=20$; these $\mathrm{K}$-means clusters are different from those defined in Fig. 5C) and for hierarchical clustering, K-means clusters 4-17 (framed in blue) were used, which showed a differential of $>0.80$ between any cell types. $(B)$ Centroids (a set of average replication-timing ratios for a given cluster) of K-means clusters 1-20 in $A$ presented in a heatmap format, which shows the average ratios for six cell states. Asterisks indicate lineage-independent EtoL clusters. (C) Sequence properties of K-means clusters $1-20$ in $A$ and $B$. Based on the average replication-timing ratios, we categorized clusters into those that stay early (EtoE), late (LtoL), middle-early (ME), middle-late (ML), shift earlier (LtoE), and shift later (EtoL). LtoE and EtoL clusters were defined using a stringent cutoff (those with a differential of $>1.0$ ). (D) Correlation between replication timing and GC/LINE-1 content in different cell states. Clusters 4-17 were used for calculation of Pearson's $R^{2}$-values. mosomes seen at the late epiblast stage, which is maintained only in ectoderm.

Altogether, at least $45 \%$ of the genome (clusters 4-17) experienced replication-timing changes at some point after the ICM stage (Fig. 6A-C). Consistent with previous studies (Hiratani et al. 2009), LtoE and EtoL changes were associated with transcriptional up- and down-regulations, respectively, in all lineages tested (Supplemental Fig. 13). Importantly, when replication-timing changes relative to the ICM stage were quantified for each cell state, a distinct set of chromosomal segments could be identified (Fig. 6C, clusters 15 and 16 , representing $6.1 \%$ of the genome or 155 megabases total) that undergo large EtoL switches ( $>1.0$ change) completed in late epiblast and maintained in all downstream lineages (Figs. 6B, 7C). We found 625 and 469 RefSeq genes within clusters 15 and 16 , respectively (Supplemental Table 1). A number of genes specifically expressed in ESCs reside in these segments (e.g., cluster 15: Fbxo15; cluster 16: Zfp42, Dppa2, Dppa4, and Morc1). By Gene Ontology (GO) analysis, cluster 15 shows significant enrichment of KRAB box and C2H2-type zinc finger genes, while cluster 16 shows significant overrepresentation of zinc finger genes, as well as cell adhesion molecules (data not shown). Many genes are still of unknown function. Taken together, results suggest that developmental replication-timing changes are widespread, affecting nearly half the genome, but involve a set of lineage-independent EtoL switches that are completed by and stably maintained after the late epiblast stage.
For further analysis, the entire set of 200-kb segments was divided into 20 groups using K-means clustering based on celltype-specific differences (Fig. 6A; Supplemental Table 2). Then, to simplify comparisons, closely related cell types were averaged, reducing the number of data sets from 17 to six, each representing a different embryonic "cell state" (Fig. 6B). DNA sequence properties within these $20 \mathrm{~K}$-means clusters (Fig. 6C) were consistent with our earlier studies (Hiratani et al. 2004, 2008). That is, the constitutively earliest (clusters 1 and 2) and latest (cluster 18-20) replicating segments were extremely GC-rich/gene-rich/LINE1-poor and GC-poor/gene-poor/LINE-1-rich, respectively, while segments subject to replication-timing changes ( $>0.80$ differential between any cell types; only clusters 4-17 met this cutoff) had intermediate GC/gene/LINE-1 content. It is these "switching" segments that determine the overall correlation of replication timing to GC/LINE-1 content, which varied between cell types but showed a sharp increase during the transition from early to late epiblast (Fig. 6D). This correlation was maintained in ectoderm, but it decreased during mesoderm and endoderm specification (Fig. 6D). Thus, the stronger alignment of replication timing to GC/LINE-1 content may represent a transient behavior of chro-
Lineage-independent early-to-late replication-timing changes are difficult to reprogram

To address the reversibility of replication-timing changes, we profiled three independent, partially reprogrammed iPSC lines (piPSCs), as well as two fully reprogrammed iPSC lines, derived from female MEF cells (Maherali et al. 2007). piPSCs are clonal cell lines that emerge from reprogramming experiments where pluripotent cells are selected based on morphology or reporter gene expression, but piPSCs fail to express many pluripotency genes. Independent piPSC clones share similar transcription profiles, suggesting reprogramming barriers at a common intermediate stage (Maherali et al. 2007; Sridharan et al. 2009). We confirmed that fully reprogrammed iPSCs (Maherali et al. 2007) have reacquired an ICM-specific profile (Fig. 7A). In contrast, piPSCs were distinct from ICM/early epiblast or early germ layer cell types, forming an independent branch upon hierarchical clustering (Fig. 7A). Remarkably, three piPSCs were highly similar, suggesting that they were indeed trapped at a similar epigenetic state despite having independent retroviral integration sites (Maherali et al. 2007). 
A

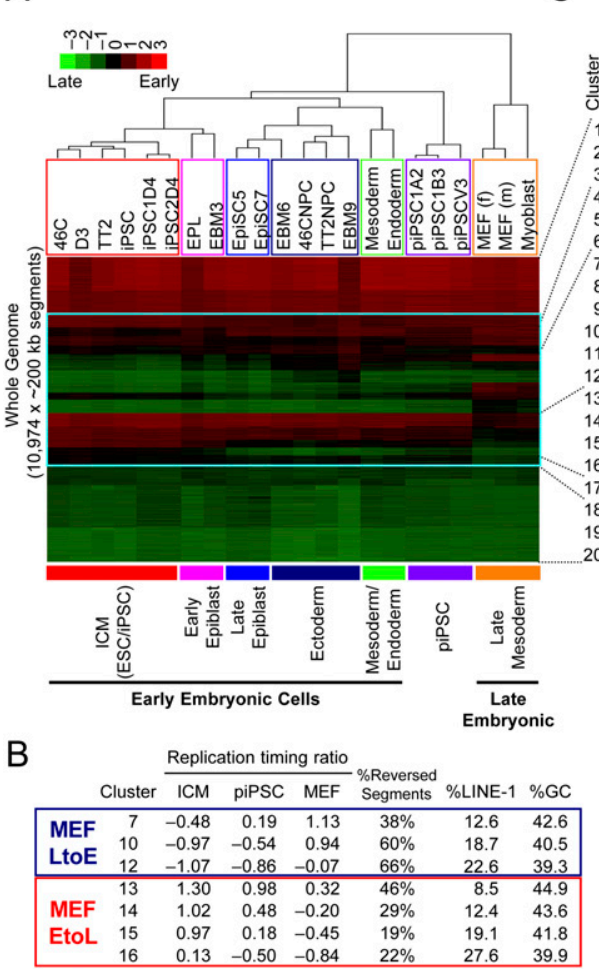

C

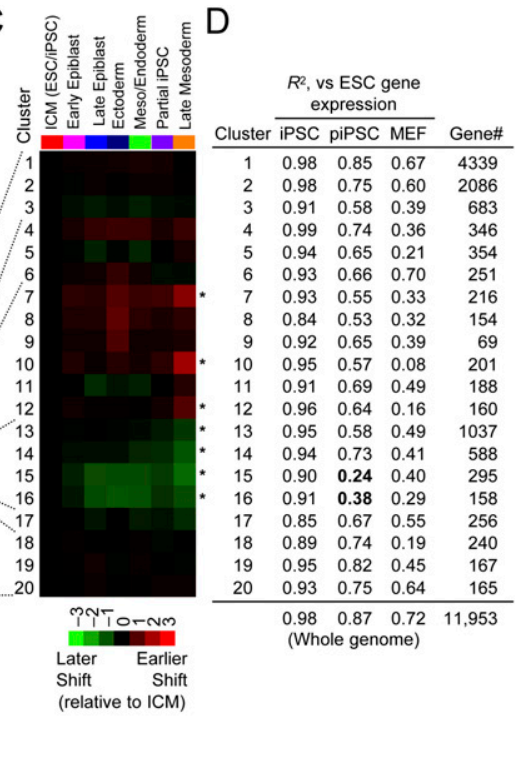

Figure 7. Lineage-independent early-to-late replication-timing changes are difficult to reprogram. (A) Hierarchical clustering of 22 cell lines, with the addition of piPSCs $(1 \mathrm{~A} 2,1 \mathrm{~B} 3, \mathrm{~V} 3)$ and iPSCs (1D4, 2D4) (Maherali et al. 2007), using K-means clusters 4-17 defined in Figure 6A (framed in blue). (B) Properties of seven K-means clusters (identical clusters as in Fig. 6A-C) that showed large differential $(>0.95)$ between MEFs and ICM. The fifth column (\% Reversed Segments) shows the difficulty of regaining ESC-specific replication timing in piPSCs derived from MEFs. The numbers represent the percentage of $200-\mathrm{kb}$ segments that showed more than $75 \%$ recovery in replication timing in piPSCs. (C) Average replication-timing differential of $\mathrm{K}$-means clusters 1-20 relative to ICM in a heatmap format (identical clusters as in Fig. $6 \mathrm{~A}-\mathrm{C}$ ). Here, red and green represent earlier and later shifts, respectively. (Asterisks) Clusters analyzed in $B$. (D) Pearson's $R^{2}$-values for pairwise comparisons of gene expression in iPSCs, piPSCs, and MEFs to ESCs within each of the 20 clusters. At the bottom of the table is the whole genome comparison. Note that clusters 15 and 16 deviate considerably from the rest of the genome when piPSCs and ESCs are compared, indicating that gene expression program in these clusters is particularly resistant to reprogramming, in contrast to other clusters and the whole genome comparison. Gene expression levels are based on the work of Sridharan et al. (2009) (Supplemental Table 2).

Focusing on segments that changed replication timing in MEFs and analyzing their replication-timing fate in piPSCs, we found that EtoL changes were more resistant to reprogramming in piPSCs, compared to LtoE changes, particularly EtoL clusters 15 and 16 (Fig. 7B, \% reversed segments). Interestingly, for EtoL changes, GC-poor/LINE-1-rich clusters (15 and 16) were the least well reversed, whereas for LtoE changes, GC-poor/LINE-1-rich clusters (10 and 12) were the easiest to be reversed (Fig. 7B). Thus, GC-poor/LINE-1-rich sequences may be more stable in a late replicating state once established, whereas GC-rich/LINE-1-poor sequences may favor an early replicating state. Clusters that changed replication timing specifically in MEFs (=late mesoderm) but not in other early embryonic cell types (Fig. 7C, clusters 10, 12, and 13) were relatively easy to reprogram (Fig. 7B, \% reversed segments), suggesting that replication-timing changes later during development are easier to reprogram than changes occurring at the epiblast stage (i.e., EtoL clusters 15 and 16). Intriguingly, when the piPSC expression profile of genes within each cluster was compared to that of ESCs, EtoL clusters 15 and 16 showed the lowest degree of correlation (Fig. 7D, $R^{2}$-values in bold; see also Supple- mental Table 3). Thus, upon reprogramming of MEFs to the piPSC state, both transcriptional activity and replication timing of segments subject to lineageindependent EtoL changes are particularly difficult to reprogram back to the ESC state.

\section{Early-to-late replication-timing} changes on autosomes and inactive $\mathrm{X}$ occur within a similar time frame

GC-poor/LINE-1-rich sequence and a lineage-independent switch to late replication are properties reminiscent of random X chromosome inactivation. Furthermore, the $\mathrm{Xi}$ undergoes a switch to late replication during late mouse epiblast development (E5.8-E6.3) (Takagi et al. 1982), while EpiSCs, which had completed the lineage-independent EtoL switches, were derived from E5.5. Although female $X$ chromosome profiles represented the average of paternal and maternal alleles, female MEF and piPSC $\mathrm{X}$ chromosome profiles exhibited replication timing clearly later than male cells or female iPSCs (Fig. 8A, cf. their color and average replication-timing ratios). This is consistent with piPSCs having failed to reactivate the Xi (Sridharan et al. 2009). We explored the temporal relationship of EtoL switches on autosomes versus the $\mathrm{Xi}$ by analyzing the female EpiSC line, EpiSC7 (Tesar et al. 2007), which had already completed autosomal EtoL changes. Although not as late replicating as female MEFs and piPSCs, the X chromosome profile in EpiSC7 did show later replication overall compared to iPSCs (Fig. 8B-G), suggesting that EtoL changes on autosomes and $\mathrm{Xi}$ are completed in a similar time frame during the late epiblast stage. The incompleteness of Xi's late replication in EpiSC7 may be related to the fact that only $44 \%$ of EpiSC7 displayed Barr body-like nuclear staining of histone H3 lysine 27 trimethylation (H3K27me3) (Fig. 8H), suggesting that the $\mathrm{X}$-inactivation process in EpiSC7 was not completed at the time of its isolation.

\section{Discussion}

In this study, we analyzed genome-wide replication-timing profiles of 15 independent mouse cell lines along with seven previously profiled cell lines (Hiratani et al. 2008). Together, these 22 cell lines represent 10 different cell types from all three germ layers during early mouse development. These data revealed extensive developmental changes in replication timing that generate cell-typespecific profiles, affect nearly half the genome, and are coordinated with transcription. Among them, we identify a distinct set of EtoL changes completed during the post-implantation epiblast stage (Fig. 9). Completion of these lineage-independent EtoL changes was coincident with repositioning of EtoL loci toward the nuclear

\section{Genome Research}


A

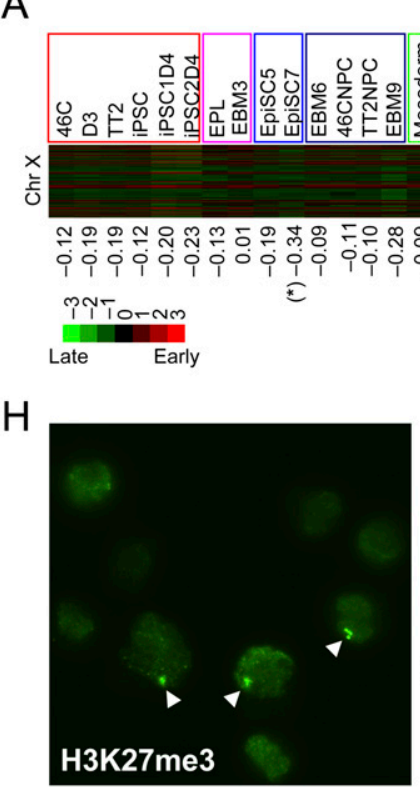

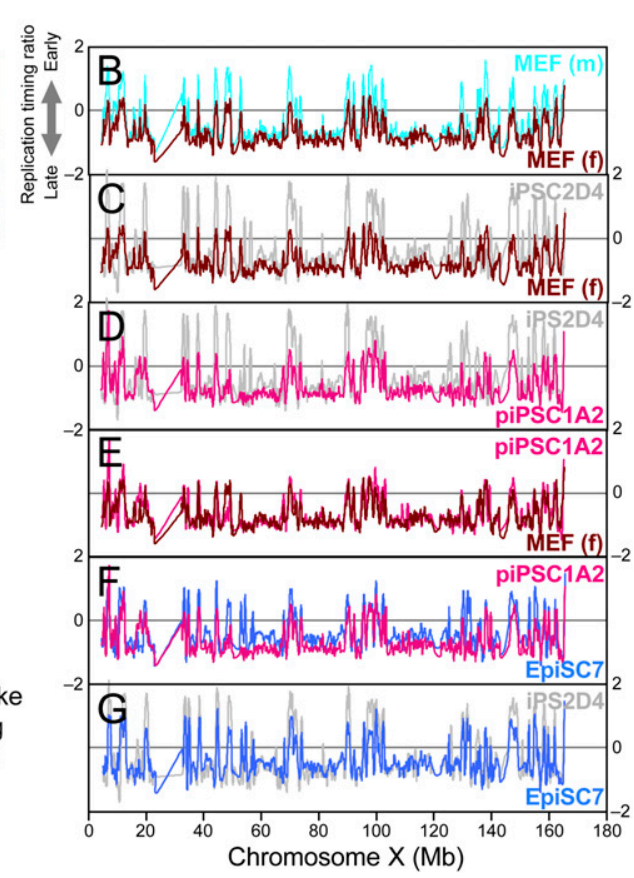

Figure 8. Analysis of late replicating inactive $X$ chromosome. (A) Heatmap representation of replication timing of all 200-kb segments along the $X$ chromosome in 22 cell lines. Below are the chromosome average replication-timing ratios. Note that piPSCs and female MEFs show markedly later replication timing (**). Female EpiSC7 is also later replicating (*). Female cell lines include iPSC1D4, iPSC2D4, EpiSC7, piPSC1A2, piPSC1B3, piPSCV3, and MEF (f); all others are male lines. $(B-G)$ Pairwise comparisons of smoothed replication-timing profiles of the $\mathbf{X}$ chromosome. Note the difference between cell types, particularly at the early replicating peaks along the chromosome. (Note: Coordinates 23-32 Mb exhibit large gaps in probes due to highly repetitive sequences.) $(H) \mathrm{H} 3 \mathrm{~K} 27$ me3 staining of EpiSC7. Forty-four percent of the cell population showed Barr body-like nuclear staining (arrowheads) of H3K27me3.

periphery and accumulation of compact chromatin near the nuclear periphery, but was prior to down-regulation of POU5F1/ NANOG/SOX2. Such regulation resembles the switch to late replication of the $\mathrm{Xi}$, which took place coincident with these lineageindependent autosomal EtoL changes. Importantly, partial iPSCs failed to restore ESC-specific replication timing and transcription programs, particularly within segments experiencing lineageindependent EtoL changes including the Xi. Taken together, our results suggest that genome-wide replication-timing profiles are a quantitative index of cell-type-specific genome organization. Furthermore, we identify a qualitative difference between replication-timing changes that occur before versus after the postimplantation epiblast stage. Changes that occur prior to the late epiblast stage are primarily lineage-independent EtoL changes that are difficult to reprogram, revealing an unanticipated epigenetic distinction between EpiSCs and earlier pluripotent cell populations. In contrast, lineage-dependent EtoL and LtoE changes occur after the late epiblast stage to create cell-type-specific profiles (Fig. 9), but these changes are relatively easier to reprogram.

Lineage-independent genome reorganization in the unspecified epiblast: “Autosomal Lyonization"

Gene expression profiles are widely accepted measures of cell fate. In fact, different embryonic and extra-embryonic cell populations during peri-implantation mouse development can be reliably distinguished by a set of established gene markers (Pfister et al. 2007). In contrast, only small differences in gene expression have been reported between ICM of the implanting blastocyst (E4.0-E4.5) and epiblast cells at E5.0 or E5.5 (Pfister et al. 2007) and yet they exhibit major phenotypic differences (Gardner and Brook 1997).
Consistent with these reports, among the 3-d intervals during neural differentiation of ESCs, the EBM3-EBM6 transition experienced the least degree of gene expression changes (Supplemental Fig. 6). Furthermore, EBM3, EPL cells, and EpiSCs shared similar expression profiles (Supplemental Fig. 8), having down-regulated a number of ESC-specific genes (e.g., Zfp42, NrOb1, and Klf4) and up-regulated epiblast genes (e.g., $F g f 5$, Dnmt3b, and Otx2). Despite small transcriptional differences, our study identified the transition from EBM3/EPL to EpiSCs, corresponding to the postimplantation epiblast stage (Fig. 9, E5.0-E6.0), as a period of lineageindependent genome reorganization. During this transition, (1) lineage-independent EtoL changes are completed; (2) correlation of replication timing to GC content is improved; (3) replicationtiming changes are accompanied by subnuclear repositioning; (4) compact chromatin emerges near the nuclear periphery; and (5) replication timing and gene expression within EtoL switching segments become difficult to reprogram back to the ESC state. These observations indicate that significant epigenetic changes can occur in the absence of large-scale transcription changes. This reorganization may represent a fail-safe means for embryos to first shut down their reversibility to ICM prior to lineage commitment. Alternatively, it may be a prerequisite for large-scale transcription changes upon lineage commitment.

Similar to the EtoL domains on autosomes, the Xi in female mammals also becomes late replicating and moves toward the nuclear periphery upon inactivation (Heard and Disteche 2006; Bartova et al. 2008), with a late-replicating $X$ chromosome emerging in the post-implantation epiblast in mice (E5.8-E6.3) (Takagi et al. 1982). Our results suggest that autosomal lineageindependent EtoL changes occur within a similar time frame in the post-implantation epiblast. Autosomal EtoL domains show a 


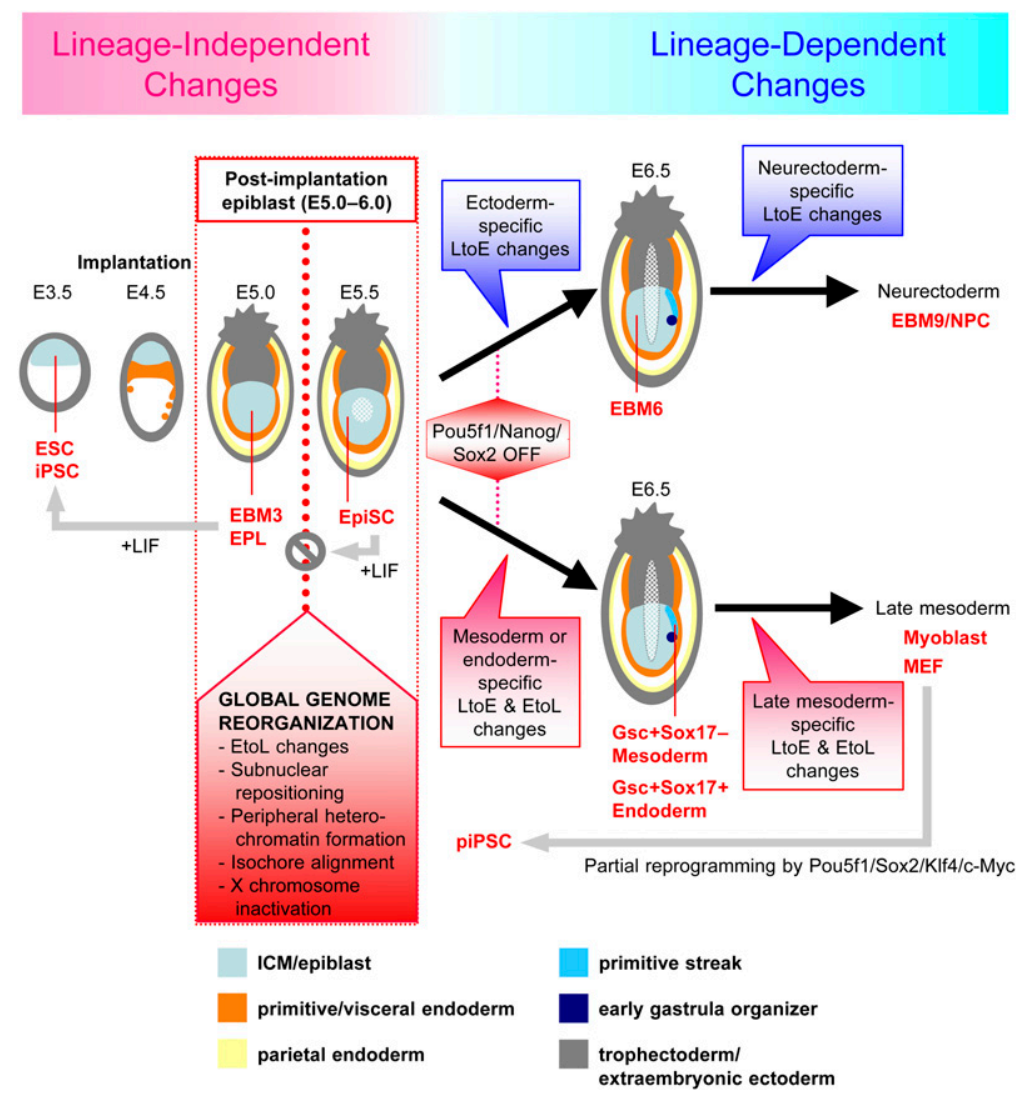

Figure 9. A model: Lineage-independent and lineage-dependent replication-timing changes before and after the post-implantation epiblast stage, respectively. Schematic diagrams of mouse embryos at different stages of embryogenesis are shown along with cell types that model different tissues and stages analyzed in this study (red). We propose that lineage-independent EtoL changes are completed by the post-implantation epiblast stage (E5.0-E6.0), prior to germ layer specification and downregulation of key pluripotency transcription factors (POU5F1/NANOG/SOX2). These changes are accompanied by several events listed, which collectively represent a form of genome reorganization ("autosomal Lyonization") that is coincident with EpiSCs having lost the ability to easily revert back to the ESC state. Lineage-dependent EtoL and LtoE changes occur continuously after the late epiblast stage to create cell-type-specific profiles. piPSCs are trapped at an epigenetic state that has not yet reprogrammed this epiblast stage genome reorganization.

tendency for transcriptional silencing, share AT/LINE-1-rich sequence features with the $\mathrm{X}$ chromosome, as well as the property of not reverting back to the ESC replication-timing state in piPSCs. Therefore, it is tempting to speculate that mechanisms regulating lineage-independent replication-timing switches on autosomes and the Xi may cross-talk in the epiblast to "Lyonize" (i.e., facultatively heterochromatinize) autosomal replication domains, as well as the Xi (Holmquist 1987). However, additional lineagespecific replication-timing changes continued to occur in more committed cell types, implying mechanisms exist that function throughout development.

\section{Loss and gain of pluripotency: An epigenetic barrier}

Recent studies have identified myriad pluripotent cell types, leading to the notion that they can adopt or fluctuate between several distinct metastable states (Chou et al. 2008, and references therein). However, the molecular basis for the intrinsic differences between metastable states and their embryonic counterpart remains poorly understood. Some of them clearly depend on different growth conditions, and yet do not show major differences in transcription profiles and histone modification patterns. Most of these cell types are distinct from, but are interchangeable with, the ESC state. For instance, subpopulations of ESCs that are negative for NANOG, ZFP42, or DPPA3 (previously known as Stella) can all easily reactivate these markers (Chambers et al. 2007; Hayashi et al. 2008; Toyooka et al. 2008). EPL cells, which can be stably maintained for multiple generations, can also readily revert back to the ESC state by culture in ESC medium containing LIF (Rathjen et al. 1999). In contrast, EpiSCs do not easily revert back to the ESC state, despite sharing the expression of key pluripotency circuitry transcription factors with ESCs and EPL cells (Brons et al. 2007; Tesar et al. 2007; Guo et al. 2009). It is believed that the molecular basis for this difficulty is epigenetic, although little is known of its nature (Hayashi et al. 2008; Guo et al. 2009).

The replication and subnuclear organization changes described above provide a plausible explanation for why it is difficult to reprogram EpiSCs back to the ESC state. Possibly, the 3D chromatin reorganization and replication-timing changes in EpiSCs represent (or reflect) an epigenetic barrier that is difficult to reprogram, similar to the female $\mathrm{Xi}$, which becomes late replicating and adopts a distinct 3D organization, the Barr body (Hiratani and Gilbert 2009). Moreover, LCP, but not HCP, genes in EtoL domains were difficult to up-regulate, suggesting a relationship of replication timing to the transcriptional responsiveness of a specific set of genes. Indeed, while iPSCs share both replication-timing and expression profiles indistinguishable from ESCs, in piPSCs, many lineage-independent EtoL autosomal segments, as well as the $\mathrm{Xi}$, were not reprogrammed back to early replication or transcriptionally reactivated.

\section{Replication profiling: A quantitative index of nuclear genome organization and stem cell identity}

Traditional differentiation of mouse ESCs to embryoid bodies generates a heterogeneous mixture of cell types and precludes the analysis of specific lineages and differentiation intermediates, making it impossible to relate the results to the normal course of embryogenesis. Using in vitro models of mouse embryogenesis, our results clearly demonstrate that replication profiling can identify differentiation intermediates that represent distinct embryonic tissue types. Furthermore, by profiling 22 cell lines representing 10 independent cell types (and seven cell states), we demonstrate that replication domain organization is cell-type-specific. Interestingly, in seven out of seven chromosomal regions examined, dynamic developmental changes in replication timing accompany subnuclear repositioning (Williams et al. 2006; Hiratani et al. 2008). 3D nuclear genome organization has long been

\section{Genome Research www.genome.org}


regarded as a form of epigenetic regulation (Gasser 2002; Fraser and Bickmore 2007; Misteli 2007). However, the idea has been plagued by the lack of a quantitative genome-wide measurement index. Although a genome-wide survey of subnuclear position is currently impractical, spatial patterns of DNA replication in the nucleus change dramatically as cells move through S-phase, demonstrating a strong correspondence of global subnuclear position to replication timing (Hiratani et al. 2009). Together, our results suggest that genome-wide replication-timing profiles are a good indicator of cell-type-specific 3D nuclear genome organization. The fact that the most dramatic reorganization occurs in the absence of global transcriptional changes yet coincides with a barrier to cellular reprogramming strengthens the case that replication domain reorganization is epigenetic by definition (Hiratani and Gilbert 2009).

\section{A key $\mathrm{G}_{1}$-phase event during post-implantation epiblast development?}

Replication-timing program is established during early $\mathrm{G}_{1}$-phase at the timing decision point (TDP), which is coincident with the anchorage of chromatin domains, reestablishing the 3D organization of chromosomes in the newly formed nucleus (Dimitrova and Gilbert 1999). Consistently, chromatin mobility is relatively high during the first 1 to $2 \mathrm{~h}$ of $\mathrm{G}_{1}$-phase, after which it is locally constrained through the remainder of interphase (Walter et al. 2003; Thomson et al. 2004). Moreover, inducible targeting of loci to the nuclear periphery requires passage through mitosis and takes place during late telophase to early $\mathrm{G}_{1}$-phase (Kumaran and Spector 2008). Our analyses suggest that replication-timing changes may need to traverse the mid-late S-phase to be accompanied by subnuclear repositioning. Together, these results predict that, during epiblast development, subnuclear repositioning takes place at the TDP within the $\mathrm{G}_{1}$-phase preceding changes in replication timing that traverse the mid-late S-phase. Mid-late S-phase is when a dramatic switch in the spatial pattern of DNA synthesis in the nucleus takes place, from an almost exclusively interior pattern during the first half of S-phase to a peripheral pattern in the second half of S-phase (Panning and Gilbert 2005; Wu et al. 2005). The post-implantation epiblast period is approximately in between E5.0 and E6.0 (Fig. 9), which presumably corresponds to less than two cell cycles (Snow 1977). Thus, we hypothesize that a key $\mathrm{G}_{1}$-phase event exists during epiblast development, in which a significantly altered subnuclear repositioning pattern is observed compared with $\mathrm{G}_{1}$ in prior cell cycles. While it is tempting to speculate the roles of chromatin modifying activities in this genome reorganization process, we and others have shown that ablation of several chromatin modifying enzymes (MLL1 [also known as MLL], MBD3, EED, SUV39H1/H2, EHMT2 [also known as G9a], DNMT1/3A/3B, DICER) show only modest effects on replication timing (Wu et al. 2006; Jorgensen et al. 2007; Hiratani et al. 2009; Yokochi et al. 2009). Moreover, the few known DNA methylation events, such as on the Pou5f1 promoter and the $\mathrm{Xi}$, are downstream (Lock et al. 1987; Feldman et al. 2006). Meanwhile, $G_{1}$-phase lengthens during the EBM3-EBM6 transition (Supplemental Fig. 14), which would provide more time for nuclei to reorganize their genome before replication initiates and could influence the reorganization process. Testing this hypothesis will require deciphering the principles of how nuclear genome reorganization occurs in early $\mathrm{G}_{1}$-phase (Hiratani et al. 2009).

\section{Methods}

\section{Cell culture and ESC differentiation}

ESC culture and differentiation of D3 ESCs to EBM9/NPC and EPL cells have been described in detail (Rathjen and Rathjen 2003). Adherent EPL cells were induced and maintained for 6-9 d in 50\% MEDII, with passages every $3 \mathrm{~d}$, before being harvested for replication profiling. EpiSC5 (clone O, male) and EpiSC7 (female) and their culture protocol have been described (Tesar et al. 2007). For mesoderm/endoderm differentiation of ES-Gsc ${ }^{\text {gfp }}$ Sox $17^{\text {huCD25 }}$ ESCs, $2-3 \times 10^{5}$ cells were seeded onto type IV collagen-coated $10-\mathrm{cm}$ dishes in SF-O3 medium (Sanko Junyaku) supplemented with $0.1 \%-0.3 \%$ bovine serum albumin, $50 \mu \mathrm{M} \beta$-mercaptoethanol, and $10 \mathrm{ng} / \mathrm{mL}$ activin A (R\&D Systems, 338-AC/CF). After $6 \mathrm{~d}$ of culture, cells were labeled with BrdU for $2 \mathrm{~h}$, immediately stained on ice with PE-conjugated anti-CD25 antibody (BD Pharmingen, 557138) to avoid cell-cycle progression, and Gsc+Sox17mesoderm and Gsc+Sox17+ endoderm cells were collected by fluorescence activated cell sorting (FACS) as described (Yasunaga et al. 2005). Sorted cells were immediately fixed in $75 \%$ ethanol and further separated into early and late $S$-phase fractions by flow cytometry for replication profiling. J185a fetal myoblast cells were grown in DMEM supplemented with $20 \% \mathrm{FBS}$ and $10 \mathrm{ng} / \mathrm{mL}$ bFGF/FGF2. Female piPSCs (1A2, 1B2), and iPSCs (1D4 and 2D4) have been described (Maherali et al. 2007). Female piPSC line V3 was derived from MEFs carrying reverse tetracycline transactivator in the ROSA26 locus (M2rtTA/M2rtTA (K Plath, unpubl.).

\section{Replication-timing profiling by microarrays}

The replication profiling protocol has been described (Hiratani et al. 2008). Sample labeling, microarray hybridization and data extraction were performed according to standard procedures by NimbleGen using a mouse whole-genome microarray with one probe every $5.8 \mathrm{~kb}$ (Roche NimbleGen Inc., 2006-07-26_MM8_ WG_CGH; 384,849 oligonucleotide probes). For all except MEFs, iPSCs, and piPSCs, two independent biological replicates were analyzed, for which early- and late-replicating DNA were labeled reciprocally with Cy3 and Cy5 (=dye switch). For MEFs, iPSCs and piPSCs, single biological replicates were profiled. However, in every case, multiple clones of the same cell type showed very high correlation, verifying the reproducibility and stability of profiles for a given cell type.

\section{Analysis of replication-timing data}

Data analyses were done using R/Bioconductor (http://www. r-project.org) and Excel (Microsoft). Data normalization, replication-timing ratio calculation of 18,679 RefSeq genes (NCBI: http://www.ncbi.nlm.nih.gov/RefSeq/), identification of replication domains by segmentation, and calculation of GC and LINE-1 content of replication domains has been described previously (Hiratani et al. 2008). Segmentation was performed as described (Hiratani et al. 2008) using parameters updated to accommodate slightly different data distributions in the differentiation intermediates. For calculating replication timing of $200-\mathrm{kb}$ segments across the genome, replication-timing ratios of nonoverlapping 35 neighboring probes were averaged $(35 \times 5.8 \mathrm{~kb}$ spacing $=201-\mathrm{kb}$ average). Thresholds for significant replication-timing changes are as indicated in the text. It is important to keep in mind that, in cases where primarily EtoL or LtoE switches predominate, there must, by definition, be many small changes throughout the rest of the genome that are below the threshold in order to balance the overall temporal order of replication. Complete replication-timing data sets for all 384,849 probes are graphically displayed and are 
available at http://www.replicationdomain.org (Weddington et al. 2008).

\section{Clustering analysis}

K-means clustering (similarity metric: Euclidian distance) and hierarchical clustering (similarity metric: centered correlation; method: average linkage) were done using Cluster 3.0 (de Hoon et al. 2004). Heatmaps and dendrograms were generated by Java TreeView (Saldanha 2004).

\section{RNA isolation, RT-PCR, and gene expression microarrays}

Total cellular RNA was isolated by RNeasy kit (Qiagen) or alternatively, RNAqueous-Micro kit (Ambion) for low-yield samples, such as mesoderm and endoderm RNA derived from cells collected by FACS. Synthesis of cDNA and RT-PCR has been described (Hiratani et al. 2004). For microarray analysis, RNA specimens were converted to double-stranded cDNA, labeled with Cy3, and hybridized according to standard procedures by NimbleGen Systems using a mouse expression microarray representing 42,586 transcripts (Roche NimbleGen Inc., 2006-08-03_MM8_60mer_expr). To avoid duplication of the same transcript within a data set, we focused on unique RefSeq genes. Among the 18,679 RefSeq genes with replication-timing ratios, 17,311 genes are present on the gene expression microarray and used for further analysis.

\section{Immunohistochemistry}

Cells were fixed with either formalin (Figs. 1, 8) or ethanol (Fig. 5). Antibody combinations were either anti-POU5F1 (BD Biosciences, 611202; 1:500) and Alexa Fluor 488 goat anti-mouse IgG (Molecular Probes, A-11029; 1:500) (Fig. 1), anti-POU5F1 (Santa Cruz, C-10; 1:50) and Alexa Fluor 594 rabbit anti-mouse IgG (Molecular Probes, A-11062; 1:200) (Fig. 5), or anti-H3K27me3 (Upstate, 07449; 1:100) and Alexa Fluor 488 goat anti-rabbit IgG (Molecular Probes, A-11008; 1:200) (Fig. 8).

\section{DNA-FISH and RNA-FISH}

DNA-FISH and RNA-FISH were performed as described previously (Hiratani et al. 2008). 3D sample fixation was employed in Figure 3 as described previously (Hiratani et al. 2008). In Figure 5D-I, 2D sample fixation (methanol:acetic acid $=3: 1$ ) was employed. For RNA-FISH, intronic regions $(0.7-1.4 \mathrm{~kb})$ were amplified by PCR with a T7 RNA promoter sequence on the $5^{\prime}$ end, which were used as template for in vitro transcription with T7 RNA polymerase. RNA products were reverse transcribed to digoxigenin (DIG)labeled, single-stranded DNA and used as RNA-FISH probes.

\section{ESI fixation, embedding, and electron microscopy}

Briefly, single-cell suspensions were prepared from ESCs or EBM3, EBM6, and EBM9 cell aggregates, applied onto a poly-Lysine coated coverslip, and fixed in $2 \%$ paraformaldehyde (EMS, RT15710) in PBS for 10 min, permeabilized in 0.5\% Triton-X 100 buffered in PBS and fixed with $1 \%$ glutaraldehyde for $5 \mathrm{~min}$. The cells were then dehydrated with ethanol in steps of 30\%, 50\%, $70 \%, 90 \%$, and $100 \%$ for $30-90$ min each. Cells were then embedded in Quetol resin. ESI electron microscopy procedures have been described (Dellaire et al. 2004).

\section{Acknowledgments}

We thank R. Didier for assistance with flow cytometry, S. Nishikawa and N. Izumi for ES-Gsc ${ }^{\text {gfp }}$ Sox $17^{\text {huCD25 }}$ ESCs and FACS protocol, N. Shindo for advice on ESC differentiation, M. Thayer for assistance with myoblast differentiation, and X. Li, J. Zhang, E. Haddock, S. Miller, and J. Shirley for technical assistance. We also thank P. Tesar for EpiSCs, as well as for helpful discussions, and the anonymous reviewers for constructive criticisms on the manuscript. This work was supported by NIH grant GM083337 (D.M.G.) and a post-doctoral fellowship from the International Rett Syndrome Foundation (I.H.). Research in the laboratory of S.D. is supported by the National Institute of Child Health and Human Development (HD049647) and the National Institute for General Medical Sciences (GM75334). Research in the laboratory of D.P.B.J. is supported by the Canadian Institutes of Health Research Operating Grant, FRN 14311.

\section{References}

Bartova E, Galiova G, Krejci J, Harnicarova A, Strasak L, Kozubek S. 2008. Epigenome and chromatin structure in human embryonic stem cells undergoing differentiation. Dev Dyn 237: 3690-3702.

Bazett-Jones DP, Li R, Fussner E, Nisman R, Dehghani H. 2008. Elucidating chromatin and nuclear domain architecture with electron spectroscopic imaging. Chromosome Res 16: 397-412.

Berezney R, Dubey DD, Huberman JA. 2000. Heterogeneity of eukaryotic replicons, replicon clusters, and replication foci. Chromosoma 108: 471-484.

Brons IG, Smithers LE, Trotter MW, Rugg-Gunn P, Sun B, Chuva de Sousa Lopes SM, Howlett SK, Clarkson A, Ahrlund-Richter L, Pedersen RA, et al. 2007. Derivation of pluripotent epiblast stem cells from mammalian embryos. Nature 448: 191-195.

Chambers I, Silva J, Colby D, Nichols J, Nijmeijer B, Robertson M, Vrana J, Jones K, Grotewold L, Smith A. 2007. Nanog safeguards pluripotency and mediates germline development. Nature 450: 1230-1234.

Chou YF, Chen HH, Eijpe M, Yabuuchi A, Chenoweth JG, Tesar P, Lu J, McKay RD, Geijsen N. 2008. The growth factor environment defines distinct pluripotent ground states in novel blastocyst-derived stem cells. Cell 135: 449-461.

de Hoon MJ, Imoto S, Nolan J, Miyano S. 2004. Open source clustering software. Bioinformatics 20: 1453-1454.

Dellaire G, Nisman R, Bazett-Jones DP. 2004. Correlative light and electron spectroscopic imaging of chromatin in situ. Methods Enzymol 375: $456-478$.

Desprat R, Thierry-Mieg D, Lailler N, Lajugie J, Schildkraut C, Thierry-Mieg J, Bouhassira E. 2009. Predictable dynamic program of timing of DNA replication in human cells. Genome Res 19: 2288-2299.

Dimitrova DS, Gilbert DM. 1999. The spatial position and replication timing of chromosomal domains are both established in early G1-phase. Mol Cell 4: 983-993.

Farkash-Amar S, Lipson D, Polten A, Goren A, Helmstetter C, Yakhini Z, Simon I. 2008. Global organization of replication time zones of the mouse genome. Genome Res 18: 1562-1570.

Feldman N, Gerson A, Fang J, Li E, Zhang Y, Shinkai Y, Cedar H, Bergman Y. 2006. G9a-mediated irreversible epigenetic inactivation of Oct-3/4 during early embryogenesis. Nat Cell Biol 8: 188-194.

Fraser P, Bickmore W. 2007. Nuclear organization of the genome and the potential for gene regulation. Nature 447: 413-417.

Gardner RL, Brook FA. 1997. Reflections on the biology of embryonic stem (ES) cells. Int J Dev Biol 41: 235-243.

Gasser SM. 2002. Visualizing chromatin dynamics in interphase nuclei. Science 296: 1412-1416.

Goren A, Cedar H. 2003. Replicating by the clock. Nat Rev Mol Cell Biol 4: 25-32.

Guo G, Yang J, Nichols J, Hall JS, Eyres I, Mansfield W, Smith A. 2009. Klf4 reverts developmentally programmed restriction of ground state pluripotency. Development 136: 1063-1069.

Hayashi K, Lopes SM, Tang F, Surani MA. 2008. Dynamic equilibrium and heterogeneity of mouse pluripotent stem cells with distinct functional and epigenetic states. Cell Stem Cell 3: 391-401.

Heard E, Disteche CM. 2006. Dosage compensation in mammals: Fine-tuning the expression of the X chromosome. Genes \& Dev 20: 1848-1867.

Hiratani I, Gilbert DM. 2009. Replication timing as an epigenetic mark. Epigenetics 4: 93-97.

Hiratani I, Leskovar A, Gilbert DM. 2004. Differentiation-induced replication-timing changes are restricted to AT-rich/long interspersed nuclear element (LINE)-rich isochores. Proc Natl Acad Sci 101: 16861-16866. 
Hiratani I, Ryba T, Itoh M, Yokochi T, Schwaiger M, Chang CW, Lyou Y, Townes TM, Schubeler D, Gilbert DM. 2008. Global reorganization of replication domains during embryonic stem cell differentiation. PLoS Biol 6: e245. doi: 10.1371/journal.pbio.0060245.

Hiratani I, Takebayashi S, Lu J, Gilbert DM. 2009. Replication timing and transcriptional control: Beyond cause and effect-part II. Curr Opin Genet Dev 19: 142-149.

Holmquist GP. 1987. Role of replication time in the control of tissue-specific gene expression. Am J Hum Genet 40: 151-173.

Jorgensen HF, Azuara V, Amoils S, Spivakov M, Terry A, Nesterova T, Cobb BS, Ramsahoye B, Merkenschlager M, Fisher AG. 2007. The impact of chromatin modifiers on the timing of locus replication in mouse embryonic stem cells. Genome Biol 8: R169. doi: 10.1186/gb-2007-8-8r169.

Karnani N, Taylor C, Malhotra A, Dutta A. 2007. Pan-S replication patterns and chromosomal domains defined by genome-tiling arrays of ENCODE genomic areas. Genome Res 17: 865-876.

Kumaran RI, Spector DL. 2008. A genetic locus targeted to the nuclear periphery in living cells maintains its transcriptional competence. J Cell Biol 180: $51-65$.

Lock LF, Takagi N, Martin GR. 1987. Methylation of the Hprt gene on the inactive X occurs after chromosome inactivation. Cell 48: 39-46.

MacAlpine DM, Bell SP. 2005. A genomic view of eukaryotic DNA replication. Chromosome Res 13: 309-326.

MacAlpine DM, Rodriguez HK, Bell SP. 2004. Coordination of replication and transcription along a Drosophila chromosome. Genes \& Dev 18: 3094-3105.

Maherali N, Sridharan R, Xie W, Utikal J, Eminli S, Arnold K, Stadtfeld M, Yachechko R, Tchieu J, Jaenisch R, et al. 2007. Directly reprogrammed fibroblasts show global epigenetic remodeling and widespread tissue contribution. Cell Stem Cell 1: 55-70.

Mikkelsen TS, Ku M, Jaffe DB, Issac B, Lieberman E, Giannoukos G, Alvarez P, Brockman W, Kim TK, Koche RP, et al. 2007. Genome-wide maps of chromatin state in pluripotent and lineage-committed cells. Nature 448: 553-560.

Misteli T. 2007. Beyond the sequence: cellular organization of genome function. Cell 128: 787-800.

Mitchell JA, Fraser P. 2008. Transcription factories are nuclear subcompartments that remain in the absence of transcription. Genes \& Dev 22: 20-25.

Norio P, Kosiyatrakul S, Yang Q, Guan Z, Brown NM, Thomas S, Riblet R, Schildkraut CL. 2005. Progressive activation of DNA replication initiation in large domains of the immunoglobulin heavy chain locus during B cell development. Mol Cell 20: 575-587.

Panning MM, Gilbert DM. 2005. Spatio-temporal organization of DNA replication in murine embryonic stem, primary, and immortalized cells. J Cell Biochem 95: 74-82.

Pelton TA, Sharma S, Schulz TC, Rathjen J, Rathjen PD. 2002. Transient pluripotent cell populations during primitive ectoderm formation: Correlation of in vivo and in vitro pluripotent cell development. J Cell Sci 115: 329-339.

Pfister S, Steiner KA, Tam PP. 2007. Gene expression pattern and progression of embryogenesis in the immediate post-implantation period of mouse development. Gene Expr Patterns 7: 558-573.

Rathjen J, Rathjen PD. 2003. Lineage specific differentiation of mouse ES cells: Formation and differentiation of early primitive ectoderm-like (EPL) cells. Methods Enzymol 365: 3-25.

Rathjen J, Lake JA, Bettess MD, Washington JM, Chapman G, Rathjen PD. 1999. Formation of a primitive ectoderm like cell population, EPL cells, from ES cells in response to biologically derived factors. J Cell Sci 112: 601-612.

Rathjen J, Haines BP, Hudson KM, Nesci A, Dunn S, Rathjen PD. 2002. Directed differentiation of pluripotent cells to neural lineages: Homogeneous formation and differentiation of a neurectoderm population. Development 129: 2649-2661.
Saldanha AJ. 2004. Java Treeview_extensible visualization of microarray data. Bioinformatics 20: 3246-3248.

Schwaiger M, Schubeler D. 2006. A question of timing: Emerging links between transcription and replication. Curr Opin Genet Dev 16: 177-183.

Schwaiger M, Stadler MB, Bell O, Kohler H, Oakeley EJ, Schubeler D. 2009. Chromatin state marks cell-type- and gender-specific replication of the Drosophila genome. Genes \& Dev 23: 589-601.

Snow MHL. 1977. Gastrulation in the mouse: Growth and regionalization of the epiblast. J Embryol Exp Morphol 42: 293-303.

Sridharan R, Tchieu J, Mason MJ, Yachechko R, Kuoy E, Horvath S, Zhou Q, Plath K. 2009. Role of the murine reprogramming factors in the induction of pluripotency. Cell 136: 364-377.

Takagi N, Sugawara O, Sasaki M. 1982. Regional and temporal changes in the pattern of X-chromosome replication during the early postimplantation development of the female mouse. Chromosoma $\mathbf{8 5}$ : 275-286.

Tesar PJ, Chenoweth JG, Brook FA, Davies TJ, Evans EP, Mack DL, Gardner RL, McKay RD. 2007. New cell lines from mouse epiblast share defining features with human embryonic stem cells. Nature 448: 196-199.

Thomson I, Gilchrist S, Bickmore WA, Chubb JR. 2004. The radial positioning of chromatin is not inherited through mitosis but is established de novo in early G1. Curr Biol 14: 166-172.

Toyooka Y, Shimosato D, Murakami K, Takahashi K, Niwa H. 2008. Identification and characterization of subpopulations in undifferentiated ES cell culture. Development 135: 909-918.

Walter J, Schermelleh L, Cremer M, Tashiro S, Cremer T. 2003. Chromosome order in HeLa cells changes during mitosis and early G1, but is stably maintained during subsequent interphase stages. J Cell Biol 160: 685-697.

Weber M, Hellmann I, Stadler MB, Ramos L, Paabo S, Rebhan M, Schubeler D. 2007. Distribution, silencing potential and evolutionary impact of promoter DNA methylation in the human genome. Nat Genet 39: 457-466.

Weddington N, Stuy A, Hiratani I, Ryba T, Yokochi T, Gilbert DM. 2008. ReplicationDomain: A visualization tool and comparative database for genome-wide replication timing data. BMC Bioinformatics 9: 530. doi: 10.1186/1471-2105-9-530.

White EJ, Emanuelsson O, Scalzo D, Royce T, Kosak S, Oakeley EJ, Weissman S, Gerstein M, Groudine M, Snyder M, et al. 2004. DNA replicationtiming analysis of human chromosome 22 at high resolution and different developmental states. Proc Natl Acad Sci 101: 17771-17776.

Williams RR, Azuara V, Perry P, Sauer S, Dvorkina M, Jorgensen H, Roix J, McQueen P, Misteli T, Merkenschlager M, et al. 2006. Neural induction promotes large-scale chromatin reorganisation of the Mash1 locus. JCell Sci 119: 132-140.

Woodfine K, Beare DM, Ichimura K, Debernardi S, Mungall AJ, Fiegler H, Collins VP, Carter NP. 2005. Replication timing of human chromosome 6. Cell Cycle 4: 172-176.

Wu R, Terry AV, Singh PB, Gilbert DM. 2005. Differential subnuclear localization and replication timing of histone H3 lysine 9 methylation states. Mol Biol Cell 16: 2872-2881.

Wu R, Singh PB, Gilbert DM. 2006. Uncoupling global and fine-tuning replication timing determinants for mouse pericentric heterochromatin. J Cell Biol 174: 185-194.

Yasunaga M, Tada S, Torikai-Nishikawa S, Nakano Y, Okada M, Jakt LM, Nishikawa S, Chiba T, Era T. 2005. Induction and monitoring of definitive and visceral endoderm differentiation of mouse ES cells. Nat Biotechnol 23: 1542-1550.

Yokochi T, Poduch K, Ryba T, Lu J, Hiratani I, Tachibana M, Shinkai Y, Gilbert DM. 2009. G9a selectively represses a class of late-replicating genes at the nuclear periphery. Proc Natl Acad Sci 106: 19363-19368.

Received August 18, 2009; accepted in revised form November 20, 2009. 


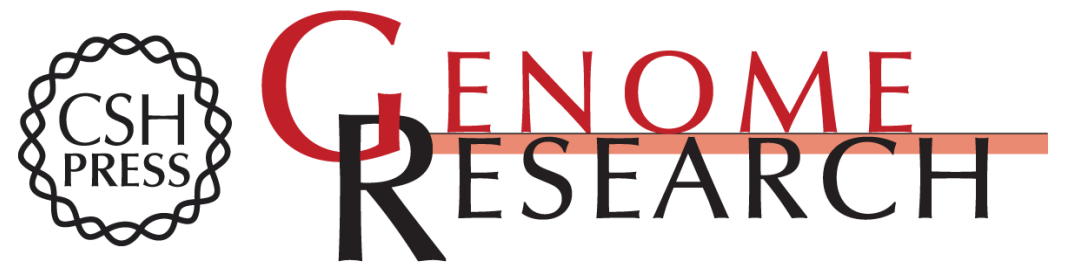

\section{Genome-wide dynamics of replication timing revealed by in vitro models of mouse embryogenesis}

Ichiro Hiratani, Tyrone Ryba, Mari Itoh, et al.

Genome Res. 2010 20: 155-169 originally published online December 1, 2009

Access the most recent version at doi:10.1101/gr.099796.109

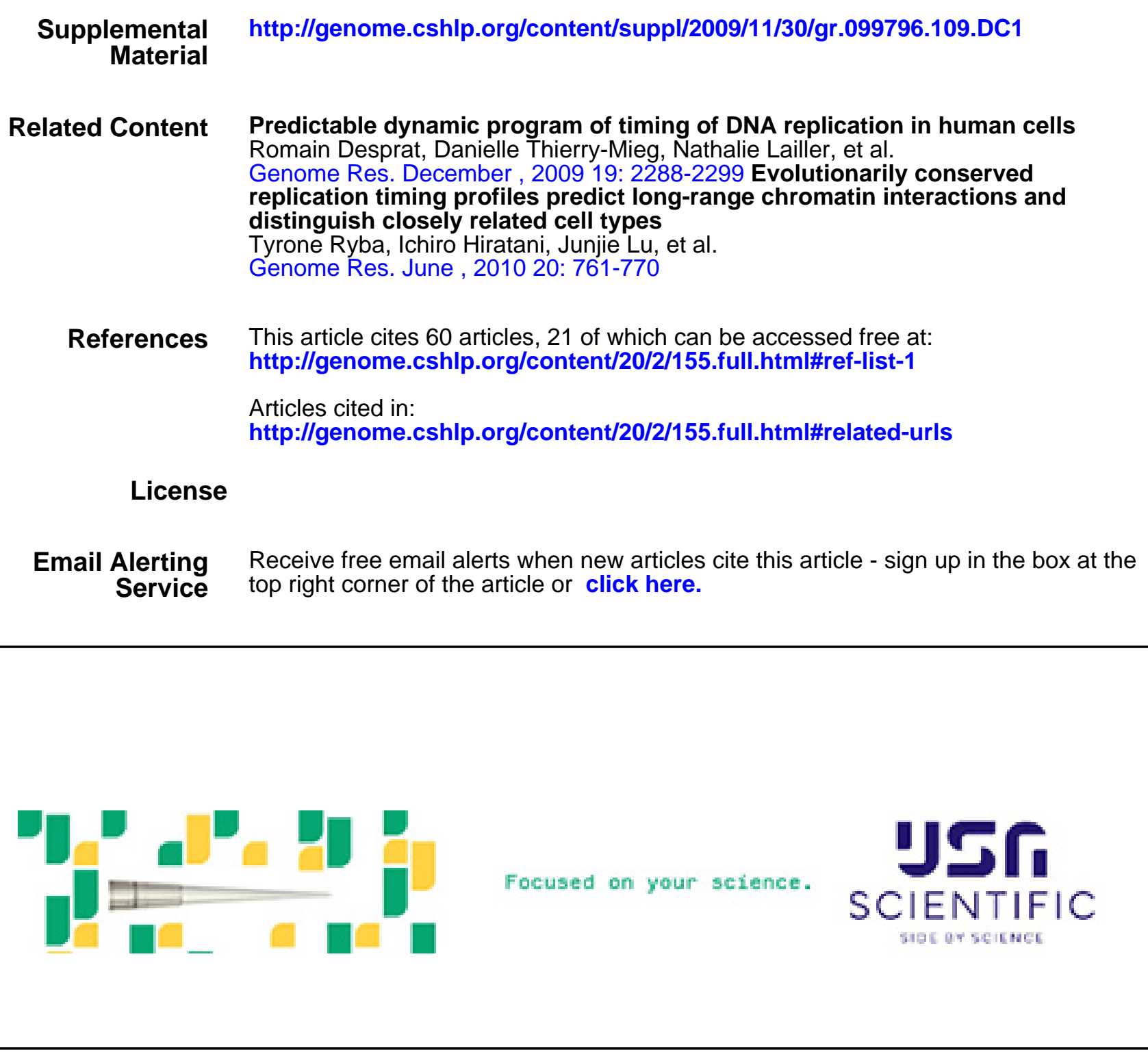

To subscribe to Genome Research go to:

https://genome.cshlp.org/subscriptions 